\title{
STOKESIAN DYNAMICS
}

\author{
John F. Brady \\ Department of Chemical Engineering, California Institute of \\ Technology, Pasadena, California 91125
}

\section{Georges Bossis}

Laboratoire de Physique de la Matière Condensée, Université de Nice, Parc Valrose, 06034 Nice Cedex, France

\section{INTRODUCTION}

Particles suspended or dispersed in a fluid medium occur in a wide variety of natural and man-made settings, e.g. slurries, composite materials, ceramics, colloids, polymers, proteins, etc. The central theoretical and practical problem is to understand and predict the macroscopic equilibrium and transport properties of these multiphase materials from their microstructural mechanics. The macroscopic properties might be the sedimentation or aggregation rate, self-diffusion coefficient, thermal conductivity, or rheology of a suspension of particles. The microstructural mechanics entails the Brownian, interparticle, external, and hydrodynamic forces acting on the particles, as well as their spatial and temporal distribution, which is commonly referred to as the microstructure. If the distribution of particles were given, as well as the location and motion of any boundaries and the physical properties of the particles and suspending fluid, one would simply have to solve (in principle, not necessarily in practice) a well-posed boundary-value problem to determine the behavior of the material. Averaging this solution over a large volume or over many different configurations, the macroscopic or averaged properties could be determined. The two key steps in this approach, the solution of the manybody problem and the determination of the microstructure, are formidable but essential tasks for understanding suspension behavior.

This article discusses a new, molecular-dynamics-like approach, which 


\section{BRADY \& BOSSIS}

we have named Stokesian dynamics, for dynamically simulating the behavior of many particles suspended or dispersed in a fluid medium. Particles in suspension may interact through both hydrodynamic and nonhydrodynamic forces, where the latter may be any type of Brownian, colloidal, interparticle, or external force. The simulation method is capable of predicting both static (i.e. configuration-specific) and dynamic microstructural properties, as well as macroscopic properties in either dilute or concentrated systems. Applications of Stokesian dynamics are widespread; problems of sedimentation, flocculation, diffusion, polymer rheology, and transport in porous media all fall within its domain. Stokesian dynamics is designed to provide the same theoretical and computational basis for multiphase, dispersed systems as does molecular dynamics for statistical theories of matter.

This review focuses on the simulation method, not on the areas in which Stokesian dynamics can be used. For a discussion of some of these many different areas, the reader is referred to the excellent reviews and proceedings of topical conferences that have appeared (e.g. Batchelor 1976a, Dickinson 1983, Faraday Discussions 1983, 1987, Family \& Landau 1984). Before embarking on a description of Stokesian dynamics, we pause here to discuss some of the relevant theoretical literature on suspensions, and dynamic simulation in general, in order to put Stokesian dynamics in perspective.

\section{Theoretical Studies and Hydrodynamic Interactions}

Theoretical approaches to suspension behavior have, for the most part, been limited to dilute concentrations at zero particle Reynolds number, where single- or two-particle interactions dominate, and have sought to determine the macroscopic properties by an expansion in number density or volume fraction. This approach dates from the original work by Einstein (1906) on the effective viscosity of a dilute suspension of spheres, and several relevant articles are available that review this area (Batchelor 1974, 1976a, Brenner 1974, Jeffrey \& Acrivos 1976, Russel 1980, Davis \& Acrivos 1985). From a predictive point of view, dilute-suspension theories are limited to low solids concentrations. Nevertheless, they have identificd many of the fundamental mechanisms operating in dispersions and provide an important foundation upon which to base further studies.

Extending dilute-suspension analyses to higher concentrations poses at least two problems. The first is the determination of the many-body interactions among the particles-particularly, the many-body hydrodynamic interactions. While extremely complex, progress can be, and has been, made in analytically computing many-body hydrodynamic interactions (Mazur \& van Saarloos 1982, van Saarloos \& Mazur 1983). These 
calculations are by no means exact for all particle-particle separations, but they do give some indication of the importance of three-body and higher order effects (Beenakker \& Mazur 1983, 1984, Beenakker 1984). In addition to many-body interactions, lubrication forces play a predominant role in determining suspension structure and behavior at high concentrations. Lubrication forces, as the name implies, result from the thin layer of viscous fluid that separates the surfaces of nearly touching particles; one of the effects of these forces is that the relative motion of particles tends to zero as the particle surfaces approach one another. Thus, to accurately model the behavior of particles in suspension, both of these important hydrodynamic effects need to be addressed.

Another aspect of the hydrodynamic interactions that causes considerable difficulty (not to mention confusion) is their long-range character. The fluid velocity disturbance caused by a particle on which a net external force acts decays as $1 / r$, where $r$ is the distance from the particle. A large collection of such forced particles, i.e. an infinite sedimenting suspension, results in a severely nonconvergent sum of interactions; the velocity of a test particle diverges as $R^{2}$, where $R$ is the size of the system. If the particles are fixed in space, as in a porous medium, rather than having a prescribed force, the long-range interactions actually change the fundamental character of the velocity disturbance caused by a particle, resulting in a screening of hydrodynamic interactions (Brinkman 1947, Tam 1969, Childress 1972, Saffman 1973, Howells 1974, Hinch 1977). Similar, but less severe, divergences occur if the particles are force-free.

The origin, significance, and interpretation of these convergence difficulties are now well understood, and several procedures have been devised for overcoming them, resulting in well-posed, absolutely convergent expressions (Batchelor 1972, Batchelor \& Green 1972, Jeffrey 1973, Hinch 1977, O'Brien 1979). Most of these methods are not, however, suitable for dynamic simulation because they preaverage the interactions. We have extended the excellent and lucid analysis of O'Brien (1979) for simulation, with the result that such diverse properties as sedimentation, permeability, and viscosity can all be treated within the same framework for any microstructural arrangement of particles. Thus, the hydrodynamic probleminherently a many-body phenomenon with singularities at large distances due to the long-range interactions and at small distances because of lubrication forces - is resolved with the Stokesian-dynamics method.

The second problem is the determination of the spatial and temporal distribution of the particles - the suspension microstructure. To date, little progress has been made that goes beyond the two-particle limit. This is due in large part to the fact that in suspensions in which particles undergo relative motion, as in response to a bulk shear flow for example, the 
distribution of particles cannot be specified a priori but rather must be found as part of the problem. Furthermore, with the exception of the Brownian-motion-dominated regime, where the equilibrium distribution (or small departures from it) can be obtained from the well-developed apparatus of statistical mechanics, most problems of interest are in regimes far from equilibrium, and there is no known way to predict the microstructure in even the simplest of problems. This aspect sets the sedimentation and viscosity problems apart from those concerned with permeability or conductivity, for in the latter the distribution of particles can be supposed known or given at the outset. Indeed, the microstructure in a flowing suspension is a dynamic rather than static entity; it determines and is determined by the bulk macroscopic motion.

The only analyses that have gone beyond the two-body limit and treated concentrated suspensions are those for spatially periodic, lattice models (Zick \& Homsy 1982, Zuzovsky et al. 1983, Nunan \& Keller 1984, Adler et al. 1985). Although the many-body problem can now be solved exactly on the unit cell, the assumption of a perfectly regular microstructure is highly restrictive. In some cases results from such analyses may not even predict qualitative behavior properly.

\section{Other Simulation Techniques}

The problems associated with the dynamical behavior of suspensions are similar to those encountered in statistical mechanics when studying the equilibrium and transport properties of condensed matter. Indeed, many analogies can be made, although they are rarely perfect. Dynamic simulation, particularly molecular dynamics, has been employed for many years, so it is natural to ask where Stokesian dynamics fits into the broader area of dynamic simulation in general.

Dynamic simulation of matter can be divided into (at least) five separate areas, distinguished by the length scale on which the phenomena are represented. On the molecular scale there is the conventional molecular dynamics of atoms and molecules pioneered by Alder \& Wainwright (1958). Here, Newton's equations of motion are simulated with particles in vacuum interacting through Lennard-Jones, hard-sphere, electrostatic, etc., types of interparticle forces. (We exclude from this discussion any systems of quantum-mechanical nature, not because molecular-dynamicslike methods do not apply, but out of ignorance.) To calculate transport properties of these atomic or molecular systems, nonequilibrium molecular dynamics, where a "macroscopic deformation" or an external field is imposed and some sort of overall constraint used to maintain a constant temperature, has gained increasing use and popularity in recent years (Hoover 1983, 1986, Evans \& Hoover 1986). 
At the next level higher in length scale, one attempts to simulate single large molecules, such as proteins or polymer chains. Here, the chemistry of the bond distances and angles plays a crucial role in generating the basic macromolecular structure. The interest is generally in understanding intramolecular aspects, such as protein folding (McCammon 1984, Hermans 1984), and those macroscopic properties, such as elasticity of glassy polymers (Theodorou \& Suter 1986), for which intrachain deformation is the dominant contribution. We designate this level as "macromolecular dynamics." For the most part in these systems, the solvent or monomer is absent, with the exception perhaps of some bound solvent in the protein problems. Thermal fluctuations are introduced (if at all) as an "add on," rather than being present as a result of a fundamental consequence of Newton's laws of motion. The "dynamics" aspect is, at present, perhaps a misnomer, but work is progressing in this area.

At the next higher level, we have entire protein or polymer molecules, or larger colloidal particles, or still larger glass beads, fibers, rods, coal particles, etc., dispersed in a continuum solvent of small molecules or particles. The physics now changes as the macroparticles interact via continuum-scale interparticle forces, such as London-van der Waals, screened electrostatic, and Derjaguin-Landau-Verwey-Overbeek (DLVO), as well as through hydrodynamic forces transmitted via the continuum fluid. If the particles are sufficiently small, the fluctuating thermal forces they receive from the fluid will influence their motion and give rise to the familiar phenomenon of Brownian motion. It is this range, where there are large particles interacting through continuum-level forces, that is the domain of Stokesian dynamics. The central elements at this level are the hydrodynamic forces, and when the particle Reynolds number is small, these are governed by the linear Stokes equations-hence the name Stokesian dynamics. Another feature of the physics at this level of description is that a suspension of interacting macroparticles does not necessarily possess a temperature. If millimeter-sized glass beads are suspended in a fluid of the same density and there is no imposed macroscopic motion, the particles will stay where they were placed initially; there is no thermal-equilibrium state.

There has been considerable work aimed at developing a simulation procedure for particles at this level of description. The pioneering work in this area was that of Ermak \& McCammon (1978) in which they treated the Brownian-motion-dominated limit, including only the simplest hydrodynamics. This work has been further developed by Dickinson and others (Dickinson 1985, Bacon et al. 1983, Dickinson et al. 1985, Ansell et al. 1985, Ansell \& Dickinson 1986a,b, Gaylor et al. 1981) and is generally known as "Brownian dynamics." Because of the incomplete treatment 


\section{BRADY \& BOSSIS}

of the hydrodynamic forces, these methods are incapable of simulating anything but the dilute Brownian limit, and we prefer the name Stokesian dynamics as more general; it includes Brownian dynamics as a special case.

Some readers may feel there should be a level between macromolecular dynamics and Stokesian dynamics for those problems, such as polymer molecules in solution, where both macroscopic hydrodynamic interactions and intramolecular chemical forces are important. Our feeling is that the physics is only well understood at the fundamental atomistic level (and therefore one must model explicitly polymer monomer segments and all the solvent molecules) or at the continuum level (and then one needs the complete hydrodynamics as in the so-called micromechanical models of polymers). To do otherwise - that is, to use some hydrodynamics and some molecular forces-is to mix physics on two different length scales, a very risky venture.

Continuing to the next higher level, one enters into the domain of "granular dynamics," where the interest is in the motion of sand grains, gravel, seeds, billiard balls, etc. While we are on a continuum scale, it is presumed that the fluid (usually air) separating the grains plays no role in the behavior of a granular flow (a high-Reynolds-number flow). The graingrain collisions dominate and, along with gravity, determine the physics. The structure of the dynamical equations is very similar to that of conventional molecular dynamics, except considerable care is needed to include the elasticity of the particles and friction, both macroscopic concepts. Like particles in a fluid, in the absence of a deforming motion, sand grains will stay where they are placed initially; there is again no a priori temperature or equilibrium state. This is a fascinating new area of dynamic simulation, and the reader is referred to the original contributions (Campbell \& Brennen 1985, Campbell \& Gong 1986, Walton et al. 1984, Walton \& Braun 1986).

The last of our five scales is gravitational or stellar dynamics. Here, planets, stars, or whole galaxies of stars are treated as point masses and interact through vacuum via Newton's law of gravity. The interest lies in questions of the stability of the solar system, the evolution of galaxies, and the large-scale structure of the Universe (Goodman \& Hut 1985).

It is interesting to trace through this changing of scales and notice that at the smallest atomic level, particles are in vacuum and interact generally through central forces. Exactly the same is true at the largest, galactic scale. At two of the intermediate scales (macromolecular dynamics and granular dynamics) the interactions are also essentially through vacuum, but they are much more complex in character. The middle (Stokesian dynamics) scale is the only one in which the continuum nature of the 
problem is fundamental and cannot be ignored. This also makes it one of the more interesting and difficult levels. Finally, we have completely ignored what is probably the most difficult scale of all-macroscopic particles in a fluid, such as sand grains in air or glass beads in water, for which the behavior of the fluid cannot be ignored (as in granular dynamics) and for which the Reynolds number for the particle motion is not small (as in Stokesian dynamics), so that the full Navier-Stokes equations need to be addressed.

With this perspective in mind, we now describe the Stokesian-dynamics method and illustrate some of its applications. These illustrations are selective and far from exhaustive; indeed, only the surface is scratched. This article is not a comprehensive review; it is an introduction to and an invitation to participate in the dynamic simulation of dispersions. It is hoped that the reader will see some possible applications of such an approach to his or her own research areas and thus will help further develop and expand this new field.

\section{SIMULATION METHOD}

The description of the Stokesian dynamics method is divided into four parts. First, the evolution equation for the suspension microstructure is presented, and second the appropriate definitions for the macroscopic properties are given. These equations are completely general, rigorous, and exact for $N$ particles suspended in a volume $V$ and show clearly the central role played by the hydrodynamic interactions. The formulation includes contributions from Brownian, interparticle, external, and hydrodynamic forces acting on the particles. In any given situation, however, not all of these forces will necessarily be present, and simplifications are possible. Owing to the linearity of the governing hydrodynamic equations, the simplifications can be obtained by simply setting the corresponding terms equal to zero. Third, a method is outlined for calculating the $N$ body hydrodynamic interactions, one that is accurate, computationally efficient, and includes both near-field lubrication forces and the dominant many-body interactions. Fourth, we show how to simulate unbounded, infinite systems, i.e. the thermodynamic limit $N \rightarrow \infty, V \rightarrow \infty$ with $N / V$ fixed. This is complicated in suspensions because of the long-range $(1 / r)$ nature of the hydrodynamic interactions, and an exact procedure is presented for solving this problem.

\section{Microstructural Mechanics}

For $N$ rigid particles suspended in an incompressible Newtonian fluid of viscosity $\eta$ and density $\rho$, the motion of the fluid is governed by the Navier- 
Stokes equations, while the particle motion is described by the coupled $N$ body Langevin equation, which can be written in the following form:

$$
\mathbf{m} \cdot \frac{d \mathbf{U}}{d t}=\mathbf{F}^{\mathrm{H}}+\mathbb{F}^{\mathrm{P}}+\mathbf{F}^{\mathrm{B}} .
$$

This equation simply states that the mass times the acceleration equals the sum of the forces. In (1) $\mathbf{m}$ is a generalized mass/moment-of-inertia matrix of dimension $6 N \times 6 N$, U is the particle translational/rotational velocity vector of dimension $6 N$, and the $6 N$ force/torque vectors $F$ represent (a) the hydrodynamic forces $\mathbf{F}^{\mathbf{H}}$ exerted on the particles due to their motion relative to the fluid, $(b)$ the deterministic nonhydrodynamic forces $\mathbf{F}^{\mathrm{P}}$, which may be either interparticle or external, and $(c)$ the stochastic forces $\mathbf{F}^{\mathbf{B}}$ that give rise to Brownian motion.

When the motion on the particle scale is such that the particle Reynolds number is small (see below for an exact specification of this condition), the hydrodynamic force exerted on the particles in a suspension undergoing a bulk linear shear flow is (Brenner \& O'Neill 1972, Kim \& Mifflin 1985, Bossis \& Brady 1984)

$$
\mathbf{F}^{\mathrm{H}}=-\mathbf{R}_{\mathrm{FU}} \cdot\left(\mathbf{U}-\mathbf{U}^{\infty}\right)+\mathbf{R}_{\mathrm{FE}}: \mathbf{E}^{\infty} .
$$

Here, $\mathbf{U}^{\infty}$ is the velocity of the bulk shear flow evaluated at the particle center (i.e. $\mathbf{U}_{\alpha}^{\infty}=\boldsymbol{\Omega}^{\infty}$ for rotation and $\mathbf{U}_{\alpha}^{\infty}=\mathbf{E}^{\infty} \cdot \mathbf{x}_{\alpha}$ for translation, where $\mathbf{x}_{\alpha}$ is the position vector of the $\alpha$ th particle); $\mathbf{E}^{\infty}(t)$ and $\boldsymbol{\Omega}^{\infty}(t)$ are, respectively, the symmetric (and traceless from continuity) and antisymmetric parts of the velocity-gradient tensor and are constant in space, but they may be arbitrary functions of time; $\mathbf{R}_{\mathrm{FU}}(\mathbf{x})$ and $\mathbf{R}_{\mathrm{FE}}(\mathbf{x})$ are the configuration-dependent resistance matrices that give the hydrodynamic force/ torque on the particles due to their motion relative to the fluid $\left(\mathbf{R}_{\mathrm{FU}}\right)$ and due to the imposed shear flow $\left(\mathbf{R}_{\mathrm{FE}}\right.$ ); and $\mathbf{x}$ is the generalized configuration vector specifying the location and orientation of all $N$ particles. The inverse of the resistance matrix $\mathbf{R}_{\mathrm{FU}}$ is known as the mobility matrix $\mathbf{M}\left(=\mathbf{R}_{\mathrm{FU}}^{-1}\right)$ and is a central element describing the hydrodynamic interactions among particles. Note that the subscripts on the matrices indicate the coupling between kinematic (U) and dynamic (F) quantities.

The deterministic, nonhydrodynamic force $\mathbf{F}^{\mathrm{P}}$ is arbitrary and may be almost any form of interparticle or external force. The stochastic or Brownian force $\mathbf{F}^{\mathrm{B}}$ arises from the thermal fluctuations in the fluid and is characterized by

$$
\left\langle\mathbf{F}^{\mathrm{B}}\right\rangle=0 \quad \text { and } \quad\left\langle\mathbf{F}^{\mathrm{B}}(0) \mathbf{F}^{\mathrm{B}}(t)\right\rangle=2 k T \mathbf{R}_{\mathrm{FU}} \delta(t) .
$$


In (3) the angle brackets denote an ensemble average, $k$ is the Boltzmann constant, $T$ is the absolute temperature, and $\delta(t)$ is the delta function. The amplitude of the correlation between the Brownian forces at time 0 and at time $t$ results from the fluctuation-dissipation theorem for the $N$-body system. On the time scales of interest, which are discussed below and are much longer than molecular times $\left(\sim 10^{-13} \mathrm{~s}\right)$, the fluctuating forces can be considered instantaneous.

The Langevin equation (1), with the hydrodynamic force given by (2) and the Brownian force by (3), is valid provided the configuration of the particles does not change significantly during the time scale of the Brownian motion, $\tau=m / 6 \pi \eta a$ (where $m$ and $a$ are the characteristic mass and size of a particle), i.e. during the time required for the particle's momentum to relax after a Brownian impulse. This condition is satisfied for most situations of interest. [See the discussions by Hinch (1975), Batchelor (1976b), Ermak \& McCammon (1978), Russel (1981), and Rallison \& Hinch (1986) for further elaboration on this point.]

The evolution equation for the particles is obtained by following Ermak \& McCammon (1978) and integrating (1) over a time step $\Delta t$ that is large compared with $\tau$ but small compared with the time over which the configuration changes. A second integration in time produces the evolution equation for the particle positions (both translational and orientational) with error of $O\left(\Delta t^{2}\right)$ :

$$
\begin{gathered}
\Delta \mathbf{x}=\operatorname{Pe}\left\{\mathbf{U}^{\infty}+\mathbf{R}_{\mathrm{FU}}^{-1} \cdot\left[\mathbf{R}_{\mathrm{FE}}: \mathbf{E}^{\infty}+\dot{\gamma}^{*-1} \mathbf{F}^{\mathrm{P}}\right]\right\} \Delta t+\nabla \cdot \mathbf{R}_{\mathrm{FU}}^{-1} \Delta t+\mathbf{X}(\Delta t), \\
\langle\mathbf{X}\rangle=0 \quad \text { and }\langle\mathbf{X}(\Delta t) \mathbf{X}(\Delta t)\rangle=2 \mathbf{R}_{\mathrm{FU}}^{-1} \Delta t .
\end{gathered}
$$

Here $\Delta \mathbf{x}$ is the change in particle position during the time step $\Delta t$ and $\mathbf{X}(\Delta t)$ is a random displacement due to Brownian motion that has zero mean and covariance given by the inverse of the resistance matrix. Also, $\mathbf{x}$ has been nondimensionalized by the characteristic particle size $a$; the time by the diffusive time scale $a^{2} / D_{0}$, where $D_{0}(=k T / 6 \pi \eta a)$ is the diffusion coefficient of a single isolated particle; the shear forces by $6 \pi \eta a^{2} \dot{\gamma}$, where $\dot{\gamma}=\left|\mathbf{E}^{\infty}\right|$ is the magnitude of the shear rate; and the interparticle forces by their magnitude $\left|\mathbf{F}^{\mathrm{P}}\right|$. The Péclet number $\mathrm{Pe}=\dot{\gamma} a^{2} / D_{0}=6 \pi \eta a^{3} \dot{\gamma} / k T$ measures the relative importance of the shear and Brownian forces, and $\dot{\gamma}^{*}=6 \pi \eta a^{2} \dot{\gamma} /\left|\mathbf{F}^{\mathrm{P}}\right|$ is a nondimensional shear rate giving the relative importance of shear and interparticle forces.

Equation (4) simply states that the motion of a particle is composed of three parts, each resulting from the basic forces in (1). There is a deterministic contribution due to the hydrodynamic shear forces $\left(\mathbf{U}^{\infty}+\mathbf{R}_{\mathrm{FU}}^{-1}\right.$ $\left.-\mathbf{R}_{\mathrm{FE}}: \mathbf{E}^{\infty}\right) \Delta t$, a contribution from the interparticle or external forces 
$\left(\mathbf{R}_{\mathrm{FU}}^{-1} \cdot \mathbf{F}^{\mathrm{P}}\right) \Delta t$, and two contributions from Brownian motion: $(a)$ a displacement due to the configuration-space divergence of the $N$-particle diffusivity $\left(\nabla \cdot \mathbf{R}_{\mathrm{FU}}^{-1}\right) \Delta t$, and $(b)$ a random step $\mathbf{X}(\Delta t)$ whose properties are such that the fluctuation-dissipation theorem is satisfied. Equation. (4) shows clearly that the suspension's behavior depends on the dimensionless parameters- $\mathrm{Pe}, \dot{\gamma}^{*}$, and $\phi$ (the volume fraction of particles). No restriction has been made to particles of identical size or shape; they need not be spherical, and if not, other dimensionless parameters characterizing their shape would be present. If more than one type of particle is present, there will then be a volume fraction $\phi_{i}$ for each type. In general, there will also be other dimensionless parameters that characterize the range, rather than the amplitude, of the interparticle forces. Other situations, such as sedimentation for example, result in similar nondimensionalizations, with a sedimentation rate setting the velocity scale rather than a shear rate. The condition that the particle Reynolds number be small will depend on the specific application. For example, in shear flow we have $\operatorname{Re}=\rho a^{2} \dot{\gamma} / \eta \ll 1$, whereas in sedimentation the condition is given by $\operatorname{Re}=\rho U_{0} a / \eta \ll 1$, where $U_{0}$ is a characteristic sedimentation velocity of an isolated particle.

If Brownian motion is unimportant, i.e. $\mathrm{Pe} \rightarrow \infty$, it is appropriate to scale time with the shear rate $\dot{\gamma}^{-1}$ rather than with the diffusive time $a^{2} / D_{0}$. This has the effect of dividing the right-hand side of (4) by $\mathrm{Pe}$, so that $\mathrm{Pe}^{-1}$ now multiplies the Brownian contributions, and they drop out in the limit $\mathrm{Pe}^{-1}=0$. The "Brownian-dynamics" simulations that have appeared in the literature (e.g. Ermak \& McCammon 1978, Dickinson 1985) are all based on the limit $\mathrm{Pe} \rightarrow 0$ and are incapable of examining the high-Pe limit because of the incomplete treatment of the hydrodynamic forces (see below). As developed here, Stokesian dynamics is completely general, encompassing both high- and low-Pe limits as special cases. Brownian dynamics is thus a subset of Stokesian dynamics.

Readers more comfortable with a continuum description of the suspension physics should know that (4) can also be obtained from a configuration-space Fokker-Planck or Smolochowski equation for the $\mathrm{N}$ particle probability distribution function $P(\mathbf{x})$, which is the appropriate level of description for times long compared with $\tau$. The conserved probability satisfies

$$
\frac{\partial P}{\partial t}+\nabla \cdot \mathbf{v} P=0
$$

The $N$-particle velocity results from the imposed flow and the forces acting on the particles, and it is given by 


$$
\mathbf{v}=\mathbf{U}^{\infty}+\mathbf{R}_{\mathrm{FU}}^{-1} \cdot\left(\mathbf{R}_{\mathrm{FE}}: \mathbf{E}^{\infty}+\mathbf{F}^{\mathrm{P}}-k T \nabla \ln P\right) .
$$

The three terms in the parentheses represent (from left to right) the velocity due to the shear, the interparticle forces, and the Brownian forces. At the Fokker-Planck level, the fluctuating Brownian forces appear as a thermodynamic force $-k T \nabla \ln P$. Time integration of (5) with (6) over a short time step $\Delta t$ results precisely in (4), showing the equivalence of the two descriptions (Ermak \& McCammon 1978).

The evolution equation (4) is the heart of the dynamic simulation. It is an exact description for $N$ particles of arbitrary size and shape suspended in a volume $V$ interacting through hydrodynamic, interparticle, external, and Brownian forces. Given an initial configuration $\mathbf{x}(0)$, Equation (4) is simply integrated in time to follow the dynamic evolution of the suspension microstructure. Note that only the initial configuration need be given, not the initial configuration and velocity as in conventional moleculardynamics-like simulations.

\section{Macroscopic Properties}

To use Stokesian dynamics to determine macroscopic properties of the suspension, corresponding average expressions must be derived. These, in general, depend on the property to be investigated, and we only discuss a few of them here. Most of the general formulas have been derived by Batchelor (1970a, 1972, 1976b, 1977), and they all involve averages of the hydrodynamic interaction tensors.

For sedimentation (relative to zero-volume-flux axes, $\mathbf{U}^{\infty}=0$; see below), the interest is in the average velocity of the particles $\langle\mathbf{U}\rangle$, where the angle brackets denote a sum over all particles, $1 / N \Sigma_{\alpha=1}^{N}$, and an average over all configurations, i.e. an average over time in the dynamic simulation. Thus, for identical particles experiencing the same external force of gravity $\mathbf{F}$, the average sedimentation velocity is given by

$$
\langle\mathbf{U}\rangle=\left\langle\mathbf{R}_{\mathrm{FU}}^{-1} \cdot \mathbf{F}\right\rangle=\langle\mathbf{M}\rangle \cdot \mathbf{F},
$$

an average of the entire mobility matrix. Generalizations of the above to unlike particles are straightforward (Batchelor 1982).

If interest is in the permeability $\mathbf{K}$ of porous media, where the particles are fixed in space and a constant average flow rate is imposed $\left(\mathbf{U}^{\infty}=\left\langle\mathbf{U}^{\infty}\right\rangle\right.$ constant), then one must determine the forces $F$ required to keep the particles fixed. The inverse of the permeability, $\mathbf{K}^{-1}$, is the "resistivity" of the porous medium and is related to the average force $\langle\mathbf{F}\rangle$ required to hold the particles fixed, i.e.

$$
\mathbf{K}^{-1}=\frac{N}{V}\left\langle\mathbf{R}_{\mathrm{FU}}\right\rangle \text {. }
$$

For diffusion, the $N$-particle diffusion tensor $\mathbf{D}$ is defined by 


\section{BRADY \& BOSSIS}

$$
\mathbf{D} \equiv k T \mathbf{R}_{\mathrm{FU}}^{-1}=k T \mathbf{M} \text {. }
$$

Several "particle diffusivities" may be defined. The short-time self-diffusivity $\mathbf{D}_{0}^{s}$, which measures the average instantaneous mobility of a particle, is given by an average over all configurations:

$$
\mathbf{D}_{0}^{s}=\left\langle\mathbf{D}_{i i}\right\rangle,
$$

where the subscript $i i$ (no sum on $i$ ) indicates that only the diagonal or self terms are included in the sum. The long-time self-diffusivity $\mathbf{D}_{\infty}^{\mathrm{s}}$, which measures the ability of a particle to wander far from its starting point, is defined as the limit as time approaches infinity of one half of the time rate of change of the mean-square position of a particle; it is given by

$$
\mathbf{D}_{\infty}^{\mathrm{s}}=\lim _{i \rightarrow \infty} \frac{1}{2} \frac{d}{d t}\left\langle\left(\mathbf{x}_{i}-\left\langle\mathbf{x}_{i}\right\rangle\right)^{2}\right\rangle .
$$

Both diffusivities are accessible by light scattering in tracer-diffusion experiments, since they are the long- and short-wavelength scattering limits, respectively (Rallison \& Hinch 1986, van Megen et al. 1986). Intermediate-time definitions are not possible, as the motion of a particle is not, in general, diffusive except at short and long times. The collectivediffusion coefficient $\mathbf{D}^{c}$, measuring particle flux in response to a concentration gradient, is closely related to sedimentation (Batchelor 1976b, 1983) and is given by

$$
\mathbf{D}^{\mathfrak{c}}=\langle\mathbf{M}\rangle \frac{\phi}{1-\phi}\left(\frac{\partial \mu}{\partial \phi}\right)_{p, T},
$$

where $\mu$ is the local chemical potential of the particles.

For rheology, the bulk stress $\langle\boldsymbol{\Sigma}\rangle$ is needed. This is defined as an average over the volume $V$ containing the $N$ particles and is given by

$$
\langle\boldsymbol{\Sigma}\rangle=\mathrm{IT}+2 \eta \mathbf{E}^{\infty}+\frac{N}{V}\left\{\left\langle\mathbf{S}^{\mathrm{H}}\right\rangle+\left\langle\mathbf{S}^{\mathrm{P}}\right\rangle+\left\langle\mathbf{S}^{\mathbf{B}}\right\rangle\right\} .
$$

Here IT stands for an isotropic term of no interest. The particles make three contributions to the bulk stress: $(a)$ a mechanical or contact stress transmitted by the fluid due to the shear flow, $\left\langle\mathbf{S}^{\mathrm{H}}\right\rangle ;(b)$ an "elastic" stress due to the interparticle forces, $\left\langle\mathbf{S}^{\mathrm{P}}\right\rangle$; and $(c)$ a direct contribution from the Brownian motion, $\left\langle\mathbf{S}^{\mathbf{B}}\right\rangle$. Note that the contributions to the bulk stress parallel the forces in the Langevin equation (1). The particle contributions to the bulk stress are given by

$$
\begin{aligned}
& \left\langle\mathbf{S}^{\mathrm{H}}\right\rangle=-\left\langle\mathbf{R}_{\mathrm{SU}} \cdot\left(\mathbf{U}-\mathbf{U}^{\infty}\right)-\mathbf{R}_{\mathrm{SE}}: \mathbf{E}^{\infty}\right\rangle, \\
& \left\langle\mathbf{S}^{\mathrm{P}}\right\rangle=-\left\langle\mathbf{x} \mathbf{F}^{\mathrm{P}}\right\rangle,
\end{aligned}
$$




$$
\left\langle\mathbf{S}^{\mathrm{B}}\right\rangle=-k T\left\langle\nabla \cdot\left(\mathbf{R}_{\mathrm{SU}} \cdot \mathbf{R}_{\mathrm{FU}}^{-\mathrm{I}}\right)\right\rangle .
$$

$\mathbf{R}_{\mathrm{SU}}(\mathbf{x})$ and $\mathbf{R}_{\mathrm{SE}}(\mathbf{x})$ are configuration-dependent resistance matrices, similar to $\mathbf{R}_{\mathrm{FU}}$ and $\mathbf{R}_{\mathrm{FE}}$, relating the particle "stresslet" $\mathbf{S}$ to the particle velocities $\mathbf{R}_{\mathrm{SU}}$ and to the imposed rate of strain $\mathbf{R}_{\mathrm{SE}}$. The particle velocities to be used in (14a) are those coming from the deterministic displacements in (4), i.e. $\mathbf{U}-\mathbf{U}^{\infty}=\mathbf{R}_{\mathrm{FU}}^{-1} \cdot\left[\mathbf{R}_{\mathrm{FE}}: \mathbf{E}^{\infty}+\dot{\gamma}^{*-1} \mathbf{F}^{\mathrm{P}}\right]$. The divergence in (14c) is with respect to the last index of $\mathbf{R}_{\mathrm{FU}}^{-1}$.

Other similar definitions will be valid for different macroscopic properties. For example, in a flocculation simulation an aggregation rate would be the appropriate macroscopic variable to calculate (Schowalter 1984). In addition to these types of macroscopic properties, all the normal particledistribution functions, such as the pair-distribution function $g(\mathbf{r})$ and the triplet-distribution function $g\left(\mathbf{r}_{1}, \mathbf{r}_{2}\right)$, can be defined and determined. Many other types of statistical properties can also be obtained, such as cluster sizes and cluster-distribution functions. One may also calculate velocity fluctuations about the average and so define a "suspension temperature," and so on. In short, since the complete microstructural dynamics is followed, all statistical properties can be determined. Note also that the $N$ particles do not have to be physically independent objects; some may be permanently linked together through the interparticle force $\mathbf{F}^{\mathbf{P}}$. The above expressions still apply and now give us information on the internal dynamics of such complex objects and the contributions to the bulk properties from these internal degrees of freedom.

We have listed the sedimentation velocity, permeability, and bulk stress in order to show the fundamental role played by the hydrodynamic resistance matrices. The entire evolution of the suspension microstructure and the macroscopically observed properties depend on these interactions, so an accurate representation of the $N$-body resistance matrices is essential. We repeat that, as written, the above equations are exact; all that remains is to approximate the hydrodynamic interactions. As mentioned in the introduction, both many-body interactions and lubrication need to be addressed. Our discussion begins with finite systems, followed by the extensions necessary for infinite systems.

\section{Hydrodynamic Interactions: Finite Particle Systems}

We have recently developed a general method to calculate the resistance matrices $\mathbf{R}_{\mathrm{FU}}, \mathbf{R}_{\mathrm{FE}}$, etc., that accounts for the near-field lubrication effects and the dominant many-body interactions (Durlofsky et al. 1987). Here, we briefly summarize the method and illustrate its accuracy with a few simple examples.

The resistance matrices $\mathbf{R}_{\mathrm{FU}}, \mathbf{R}_{\mathrm{FE}}, \mathbf{R}_{\mathrm{SU}}$, and $\mathbf{R}_{\mathrm{SE}}$ can be written as part 
of a "grand resistance" matrix $\mathscr{R}$, which relates the force/torque (F) and stresslet (S) exerted by the fluid on the particles to the particle velocities and the rate of strain:

$$
\begin{aligned}
& \left(\begin{array}{l}
\mathbf{F} \\
\mathbf{S}
\end{array}\right)=-\mathscr{R} \cdot\left(\begin{array}{c}
\mathbf{U}-\mathbf{U}^{\infty} \\
-\mathbf{E}^{\infty}
\end{array}\right), \\
& \mathscr{R}=\left(\begin{array}{ll}
\mathbf{R}_{\mathrm{FU}} & \mathbf{R}_{\mathrm{FE}} \\
\mathbf{R}_{\mathrm{SU}} & \mathbf{R}_{\mathrm{SE}}
\end{array}\right) .
\end{aligned}
$$

The corresponding inverse or "grand mobility" matrix $\mathscr{M}$ representation is

$$
\left(\begin{array}{c}
\mathbf{U}-\mathbf{U}^{\infty} \\
-\mathbf{E}^{\infty}
\end{array}\right)=-\left(\begin{array}{ll}
\mathbf{M}_{\mathrm{UF}} & \mathbf{M}_{\mathrm{US}} \\
\mathbf{M}_{\mathrm{EF}} & \mathbf{M}_{\mathrm{ES}}
\end{array}\right) \cdot\left(\begin{array}{l}
\mathbf{F} \\
\mathbf{S}
\end{array}\right)
$$

For just two spherical particles the grand resistance and grand mobility matrices are known exactly for all center-center separations. For $N$ particles, short of solving the full $N$-body problem, we need to make some approximations. The method we have developed exploits the fact that the lubrication effects are most conveniently treated in the resistance formulation, whereas many-body interactions are more easily incorporated in the mobility formulation.

Starting from the integral solution for Stokes flow, in conjunction with Faxén laws for particle velocities, we form the $N$-sphere grand mobility matrix by expanding the force density on the surface of each particle in a series of moments. The zeroth moment is just the total force $\mathbf{F}$, while the first moment has both antisymmetric and symmetric parts (the torque $\mathbf{L}$ and stresslet $\mathbf{S}$, respectively). Thus, each particle is represented by its first few multipoles. In this moments expansion, the part of the mobility matrix $\mathbf{M}_{\mathrm{UF}}$ coupling translational velocities and forces is the well-known RotnePrager tensor. The other matrices are of similar structure. The first neglected term in this moments expansion comes from the quadrupole densities of the particles, and, since they are induced, the leading error in $\mathbf{M}_{\mathrm{UF}}$ is $O\left(1 / r^{6}\right)$, where $r$ is a characteristic interparticle spacing. [The errors to the other mobility matrices in (17) are of even higher order.] Both $\mathscr{R}$ and $\mathscr{M}$ and the matrices $\mathbf{R}_{\mathrm{FU}}, \mathbf{R}_{\mathrm{SE}}, \mathbf{M}_{\mathrm{UF}}$, and $\mathbf{M}_{\mathrm{ES}}$ are symmetric and positive definite.

Once constructed, the grand mobility matrix, denoted $\mathscr{M}^{\infty}$, is inverted to yield a far-field approximation to the grand resistance matrix of (16). While the mobility matrix is pairwise additive, inverting it solves the manybody problem at the level of forces and stresslets; thus, the resistance matrix is a true many-body interaction. The inversion of the mobility matrix reproduces both the screening characteristic of a porous medium 
(which is the nature of a two-particle interaction in $\mathbf{R}_{\mathrm{FU}}$, since all other particles are presumed fixed) and the effective viscosity of free suspensions.

This many-body approximation to the resistance matrix still lacks, however, lubrication, which would only be reproduced upon inversion of the mobility matrix if all multipole moments were included. Because of their short-range nature, lubrication forces are essentially two-body interactions, and we introduce them in a pairwise-additive fashion in the resistance matrix. To each element of $\left(\mathscr{M}^{\infty}\right)^{-1}$ we add the known exact twosphere resistance interactions (Arp \& Mason 1977, Jeffrey \& Onishi 1984, Kim \& Mifflin 1985), which we designate as $\mathscr{R}_{2 \mathrm{~B}}$ (for two-body resistance matrix). However, the far-field parts of the two-sphere resistance interactions have already been included upon the inversion of $\mathscr{M}^{\infty}$. Thus, in order not to count these interactions twice, we must subtract off the two-body interactions already included in $\left(\mathscr{M}^{\infty}\right)^{-1}$, which are found by inverting a two-sphere mobility matrix to the same level of approximation as in $\mathscr{M}^{\infty}$. Denoting this resistance matrix as $\mathscr{R}_{2 \mathrm{~B}}^{\infty}$, our approximation to the grand resistance matrix that includes near-field lubrication and farfield many-body interactions is

$$
\mathscr{R}=\left(\mathscr{M}^{\infty}\right)^{-1}+\mathscr{R}_{2 \mathrm{~B}}-\mathscr{R}_{2 \mathrm{~B}}^{\infty} \text {. }
$$

The grand resistance matrix is then partitioned as in (16) and used in both the evolution equation (4) and the calculation of the macroscopic properties (7)-(14).

This procedure captures both the near- and far-field physics and has given excellent results for all situations in which a comparison has been possible. As an example, in Figure 1 we compare the drag coefficient $\lambda$ (defined as $\lambda=F / 6 \pi \eta a U$, where $F$ is the external force) for a horizontal chain of seven identical, uniformly spaced spheres settling due to gravity at three different interparticle spaces calculated by Stokesian dynamics, with the results of Ganatos et al. (1978), which should be considered the exact solution. Results are only shown for half of the spheres in the chain because the drag coefficient is symmetric about the central sphere for chains with an odd number of spheres. Computation times are a factor of $10^{3}$ times faster with Stokesian dynamics. For a complete discussion of the comparisons made, see Durlofsky et al. (1987).

To illustrate the importance of the lubrication forces and the fact that they are maintained only through the resistance formulation, consider the following simple example. Given a chain of closely spaced particles, with a force directed along the chain axis applied to one particle at the end, the entire chain will move as if it were a rigid rod. The particles themselves never need come into contact, nor do they need to exert any interparticle nonhydrodynamic forces on one another. Lubrication alone suffices to 


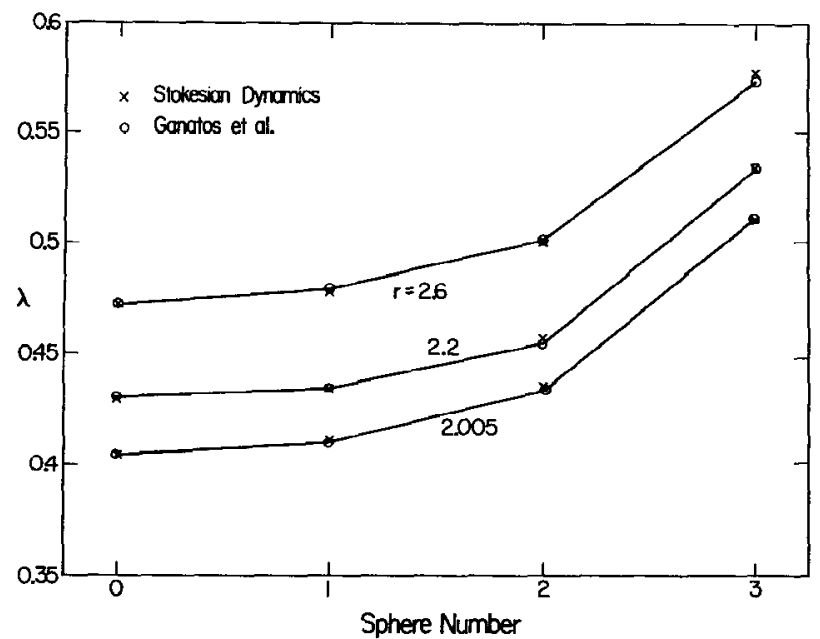

Figure 1 Comparison of the drag coefficient $\lambda=F / 6 \pi \eta a U$ for horizontal chains of seven sedimenting spheres $(X)$ with the numerical results of Ganatos et al. (1978) (O). The sphere center-center spacing is varied: $r=2.6,2.2$, and 2.005. Only half the chain is shown, as $\lambda$ is symmetric about the central sphere.

move the entire chain. Indeed, a chain of many particles should behave as a slender body, and the translational velocity can be calculated from slender-body theory (Chwang \& Wu 1975). For a spheroidal particle subjected to a total force $F$, the translational velocity in the direction of the chain axis is given by

$$
U^{S B}=\frac{F}{6 \pi \eta a} \frac{3}{2} \frac{\ln (2 l / a)-1 / 2}{N}\left[1+O\left(\frac{a}{l}\right)^{2}\right]
$$

where $a$ and $l$ are the half-lengths of the minor and major axis, respectively $(a / l \ll 1)$, and where for a chain of $N$ spheres, we have $l / a=N$. The factor of $N$ in the denominator is needed because the force $F$ was applied to only one particle, not to all $N$ particles. Figure 2 shows a comparison of $U$ obtained by Stokesian dynamics and the above expression $U^{\mathrm{SB}}$ obtained from slender-body theory; the ratio $U / U^{\mathrm{SB}}$ is plotted vs. $N$. Such an effect cannot be captured using a pairwise-additivity approximation in the mobility formulation.

Results for a slightly different slender-body problem are also shown in Figure 2. Here, we have a linear chain of force- and torque-free spheres along the compressive axis of a linear shear flow. The relevant quantity is the stresslet of the rod, which for spheroidal rods has the form (Batchelor 1970b) 


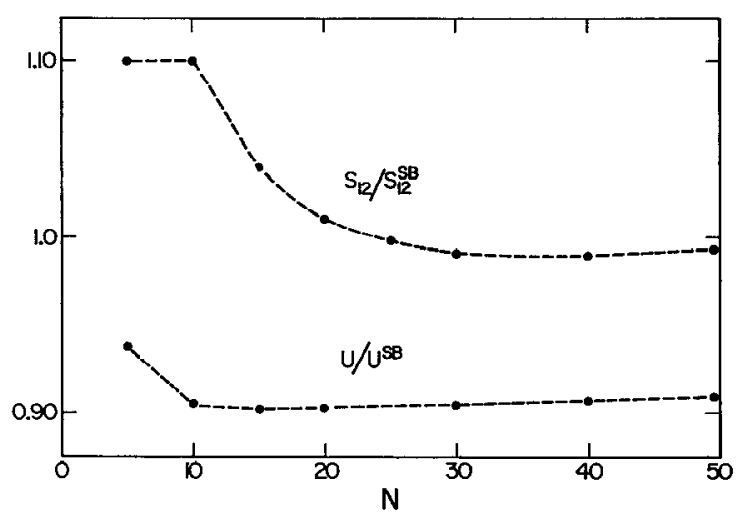

Figure 2 Comparison of the translational velocity $U$ and stresslet $S_{12}$ for a linear chain of $N$ equally spaced spheres at a center-center separation of $2+10^{-5}$ with the results of slenderbody theory ( $U^{\mathrm{SB}}$ and $S_{12}^{\mathrm{SB}}$, respectively).

$$
S_{12}^{\mathrm{SB}}=\frac{20}{3} \pi \mu a^{3} E_{12} \frac{1}{5} \frac{(l / a)^{3}}{\ln (2 l / a)-3 / 2}\left[1+O\left(\frac{a}{l}\right)^{2}\right] .
$$

Again, the agreement is seen to be excellent. This is actually quite remarkable, because a chain of spheres is not a spheroidal particle and only the correct scaling with $N$ is to be expected. If the lubrication forces were not modeled correctly in this example (i.e. if a pairwise additivity of velocities were used), then at the next time step in a dynamic simulation, particles would overlap with one another-an obviously aphysical result.

The method we have outlined accurately simulates the behavior of a finite number of particles in an unbounded Stokes flow. In addition to its application to dynamic simulation, the method can also be used as a "computational engine" to generate hydrodynamic data that may be useful in applications other than simulation. While accurate, the method can systematically be improved by including additional moments in the expansion or by directly solving the integral equation for Stokes flow; either improvement, however, results in an increase in computation time as the number of degrees of freedom per particle increases from the 11 needed here.

If the mobility matrix $\mathbf{R}_{\mathrm{FU}}^{-1}$ could be constructed directly, the calculations would ideally only require $O\left[(6 N)^{2}\right]$ operations. By the introduction of lubrication into the resistance matrix, our method requires an $O\left[(6 N)^{3}\right]$ solution, and to obtain many-body interactions it requires an $O\left[(11 N)^{3}\right]$ inversion. If Brownian motion is present, however, the random displacements $\mathbf{X}(\Delta t)$ in (4) require taking the square root of the mobility 


\section{BRADY \& BOSSIS}

matrix, which is in itself an $O\left[(6 N)^{3}\right]$ operation. Thus, it seems that the hydrodynamic interactions are inherently an $O\left(N^{3}\right)$ problem. As a final note, since variations in the grand mobility matrix occur when the particle separation has changed an amount of order the particle size, whereas variations in the grand resistance matrix occur when separation changes are of order the interparticle spacing, a multiple time-stepping procedure can be used with the mobility matrix inverted infrequently.

\section{Hydrodynamic Interactions: Infinite Suspensions}

The long-range nature $(1 / r)$ of the hydrodynamic interactions requires care in simulating infinite suspensions (i.e. in letting $N \rightarrow \infty, V \rightarrow \infty$, and keeping $N / V$ fixed). A simple summation of interactions results in badly divergent expressions. While there are several alternate ways to overcome this convergence problem, only the method presented by O'Brien (1979) can be used in dynamic simulation. We briefly outline here an extension we have made to this method; a more detailed development is in J. F. Brady et al. (submitted for publication). We only discuss the lowest level of point forces; once the idea is understood, extensions to include finitesize particles with torques, stresslcts, etc., are straightforward.

In O'Brien's method we start from an integral representation for the solution to the Stokes equations for the velocity field $\mathbf{u}(\mathbf{x})$ at a point $\mathbf{x}$ in the fluid in terms of $(a)$ integrals of the force distribution on the surfaces of the particles and $(b)$ an integral over a mathematical surface $\Gamma$ of large radius that cuts through both the fluid and the particles:

$$
\mathbf{u}(\mathbf{x})=-\frac{1}{8 \pi \eta} \sum_{\alpha=1}^{N} \int_{S_{\alpha}} \mathbf{J} \cdot \boldsymbol{\sigma} \cdot \mathbf{n} d S-\frac{1}{8 \pi \eta} \int_{S_{1}}[\mathbf{J} \cdot \boldsymbol{\sigma}+\mathbf{K} \cdot \mathbf{u}] \cdot \mathbf{n} d S .
$$

Here, $\mathbf{J}$ is the Green function for Stokes flow, $\mathbf{J}=\left(\mathbf{I}+\mathbf{r r} / r^{2}\right) / r, \mathbf{K}=$ $-6 \eta \mathbf{r r r} / r^{5}, \mathbf{I}$ is the unit isotropic tensor, $\mathbf{r}=\mathbf{x}-\mathbf{y}, \mathbf{y}$ is a point on the surface, $\boldsymbol{\sigma}$ is the fluid-stress tensor, and $\mathbf{n}$ is the outer normal to the surfaces (i.e. pointing into the volume $V$ containing the $N$ particles). In the sum, only the particle surfaces within $\Gamma$ are included.

Equation (19) is an exact formulation for rigid particles. No divergences occur because we have a finite region bounded by the surface $\Gamma$. This surface is an arbitrary one immersed in an unbounded, statistically homogeneous suspension, i.e. the suspension continues outside the surface $\Gamma$. If the radius of this surface is taken to be very large, the variation in $\mathbf{J}$ and $\mathbf{K}$ will be small over a surface element $d S_{\Gamma}$ that cuts many particles and the fluid. Thus, in the integrand of the second integral we may replace $\sigma$ and $\mathbf{u}$ by their suspension averages - fluid- and particle-phase averages$\langle\boldsymbol{\sigma}\rangle$ and $\langle\mathbf{u}\rangle$. This is the key step, and the only assumption made, in 
O'Brien's method. In a statistically homogeneous suspension, $\langle\boldsymbol{\sigma}\rangle$ and $\langle\mathbf{u}\rangle$ are either constants or linear functions of position that arise from the average pressure in $\langle\boldsymbol{\sigma}\rangle$ and a linear shear flow in $\langle\mathbf{u}\rangle$.

Using the divergence theorem and introducing the suspension-average quantities, we can manipulate (19) to give

$$
\mathbf{u}(\mathbf{x})-\langle\mathbf{u}(\mathbf{x})\rangle=\frac{1}{8 \pi \eta} \sum_{\alpha=1}^{N} \mathbf{J}\left(\mathbf{x}-\mathbf{x}_{\alpha}\right) \cdot \mathbf{F}^{\alpha}-\frac{n}{8 \pi \eta} \int_{0}^{R} \mathbf{J} \cdot\langle\mathbf{F}\rangle d V .
$$

Here $\mathbf{F}^{\alpha}=-\int_{S_{\alpha}} \boldsymbol{\sigma} \cdot \mathbf{n} d S$ is the force that the $\alpha$ th particle exerts on the fluid, $\langle\mathbf{F}\rangle$ is the average force, $n=N / V$ is the number density of particles, and $R$ is the radius of the volume $V$ enclosed by the surface $\Gamma$. The above reduction is valid for point forces only, but it is straightforward to generalize to the complete problem. It is now permissible to let $R \rightarrow \infty$, because at large distances from $\mathbf{x}$ the sum becomes equivalent to a volume integral of $n$ times the average force, and (20) results in a finite convergent expression for $\mathbf{u}-\langle\mathbf{u}\rangle$. Physically, the integral represents a "back flow" of fluid, relative to zero-volume-flux axes $\langle\mathbf{u}\rangle=0$, caused by the macroscopic pressure gradient that balances the excess weight, $\langle\mathbf{F}\rangle \neq 0$, of the particles. It is the velocity relative to this average back flow that is the physically significant quantity, not its absolute value.

Using the above procedure, in conjunction with Faxén laws for the particle velocities, an exact, absolutely convergent expression for the particle velocities can be constructed. No assumptions have been made about the distribution of particles within $V ;(20)$ applies equally well to periodic lattices and random suspensions. Continuing in this way for the torques, rotational velocities, stresslets, and rate of strain, as well as including the finite size of the particles, we develop a convergent representation for the grand mobility $\mathscr{M}$ of (17). $\mathbf{U}^{\infty}$ and $\mathbf{E}^{\infty}$ in (17) must now be interpreted as the suspension-average velocity and rate of strain evaluated at the center of particle $\alpha$, and there will appear volume integrals of the average force $\langle\mathbf{F}\rangle$, torque $\langle\mathbf{L}\rangle$, and stresslet $\langle\mathbf{S}\rangle$, analogous to that appearing in (20).

While completely general and correct, $(20)$ is not yet in a form suitable for simulation. The sum and integral in (20) both increase with $R$, but their difference is finite and is what is needed. Also, many particles may be needed before the sum approximates a continuous distribution and convergence is obtained. In order to reduce the number of particles needed and to accelerate the convergence of expressions like (20), we take a finite number $N_{1}$ of particles and replicate them periodically within the volume $V$. Thus, for the velocity of particle $\alpha$ at the center of its periodic cell, Equation (20) becomes

$$
\mathbf{U}^{\alpha}-\left\langle\mathbf{u}\left(\mathbf{x}_{\alpha}\right)\right\rangle=\frac{1}{8 \pi \eta} \sum_{y} \sum_{\beta=1}^{N_{1}} \mathbf{J}\left(\mathbf{x}_{\alpha}-\mathbf{x}_{\beta}\right) \cdot \mathbf{F}^{\beta}-\frac{n}{8 \pi \eta} \int_{0}^{\infty} \mathbf{J} \cdot\langle\mathbf{F}\rangle d V,
$$


where $\gamma$ labels the periodic cells and the prime on the sum indicates that for $\alpha=\beta$ in cell $\gamma=1, \mathbf{J}$ is replaced by $\mathbf{I}$, giving the correct self-term.

If $N_{1}$ were sufficiently large, then the contribution to the $\alpha$ th particle velocity from particles in cells $\gamma>1$, outside its own periodic box, would cancel the part of the integral from $L$ to $\infty$, where $L$ is the size of a periodic box. There would remain, however, a constant from the back-flow integral from 0 to $L$. Because of the slow covergence of the difference between the discrete sum and the continuous integral, $N_{1}$ may need to be prohibitively large. Expressions of this type containing so-called lattice sums occur frequently in electrostatic problems (for example, in computing the cohesive energy, or Madelung constant, of an ionic crystal) and can be accelerated using a method due to Ewald (1921), which rewrites the sum into two rapidly converging parts, one in real space and the other in reciprocal space.

Beenakker (1986) has recently worked out the Ewald sums for both $\mathbf{J}$ and the more complete Rotne-Prager tensor used in $\mathbf{M}_{\mathrm{UF}}$ of (17) under the assumption that the average force $\langle\mathbf{F}\rangle$ is zero, i.e. only the sums in (21) are used, with no back-flow integral. Mathematically, stipulating that $\langle\mathbf{F}\rangle=0$ removes a singular term from the reciprocal lattice sum at $\mathbf{k}=\mathbf{0}$, where $\mathbf{k}$ is a reciprocal lattice vector. When the average force is not zero, however, the back-flow integral obtained from O'Brien's method precisely cancels this singular term at $\mathbf{k}=0$, and Beenakker's Ewald-summed Rotne-Prager tensor (which we denote by $\mathbf{M}_{\mathrm{UF}}^{*}$ ) is correct whether or not there is a nonzero average force on the particles. This may appear to be a rather surprising result, but there is a simple intuitive argument that shows that it must be true. The mobility matrix is a purely geometric quantity that describes interactions among particles. It cannot depend on the velocities, forces, stresslets, etc., that the particles ultimately have. Therefore, it must be the same whether or not the average force is zero. In other words, when we write the mobility matrix in (17), the particles do not know whether the forces sum to zero or to a finite average; the particle interactions must be the same in the two cases.

Continuing in this manner-O'Brien's method and Ewald sums-for all the divergent or conditionally convergent interactions, we construct a new grand mobility matrix $\mathscr{M}^{*}$ in place of $\mathscr{M}^{\infty}$ for use in (17). This joins nicely with our moments expansion because the stresslet-rate of strain interactions, $\mathbf{M}_{\mathrm{ES}}$, decay as $r^{-3}$ and are conditionally convergent. There is one other conditionally convergent piece, however, that needs to be included: a mean-field quadrupole term. Although the quadrupoles are induced, in a suspension of forced particles $(\langle\mathbf{F}\rangle \neq 0)$ each particle has an average induced quadrupole moment of magnitude $\phi\langle\mathbf{F}\rangle$. If we replace 
each particle's induced quadrupole by the average, the contribution the quadrupoles make to the particle velocities can be conveniently included in $\mathbf{M}_{\mathrm{UF}}^{*}$. With this mean-field quadrupole approximation, $\mathscr{M}^{*}$ replaces $\mathscr{M}^{\infty}$ in (18) and lubrication is added in as before, because these contributions are all short ranged.

A simulation proceeds by constructing $\mathscr{M}^{*}$ as described above and using conventional or sheared periodic boundary conditions (Bossis \& Brady $1984)$ for the short-range lubrication interactions via (18). Note also that the macroscopic properties (7-14) are found after $\mathscr{R}$ has been partitioned, and thus they too are rendered convergent. It should also be appreciated that the Ewald sums and the periodic boundary conditions are a computational convenience; they accelerate the convergence of (20) and allow fewer particles, $N_{1}$, to be used, but they are not essential. They do, however, introduce long-range periodicity into the simulations, and in order to model unbounded disordered media, some care is needed to insure that the periodicity does not cause unwanted effects. This can be assured by requiring that the local structural length scales are all smaller than the size of the periodic cell. Rendering the expressions convergent through proper treatment of the physics by O'Brien's method is the fundamental step. Note also that in using O'Brien's method to overcome the convergence problems, one does not rely on the moments expansion; the method is equally valid if one solves the full integral equation for Stokes flow.

To illustrate that the above procedure is correct and accurate, we compare results obtained using it with the only exact results for many-body systems - spatially periodic suspensions. While the periodicity is a highly restrictive microstructure, it does allow an exact calculation and provides for a convenient test case. In Figure 3 we compare the results for the sedimentation velocity [cf. Equation (7)] of a simple cubic lattice of identical spheres as a function of volume fraction $\phi$. The solid curve is the Stokesiandynamics result; the dashed curve is the result of Zick \& Homsy (1982), who accurately solved the integral equation for Stokes flow using the periodic Green function; and the dotted curve is the point-force solution of Saffman (1973). The agreement is quite good, from the dilute limit up to close packing. In order to facilitate comparison at low and high $\phi$, two different scales are used in the ordinate and abscissa. At low $\phi$ the exact and Stokesian-dynamics curves are indistinguishable. Because there are relatively large channels in the periodic sedimenting array through which the upwardly flowing fluid may pass, lubrication forces do not enter and their inclusion in the resistance matrix has little effect on the results. Hence, this explains the less-than-precise agreement for $\phi>0.3$. At low $\phi$ the agreement is not just good, it is exact; for point forces only, the Ewald- 


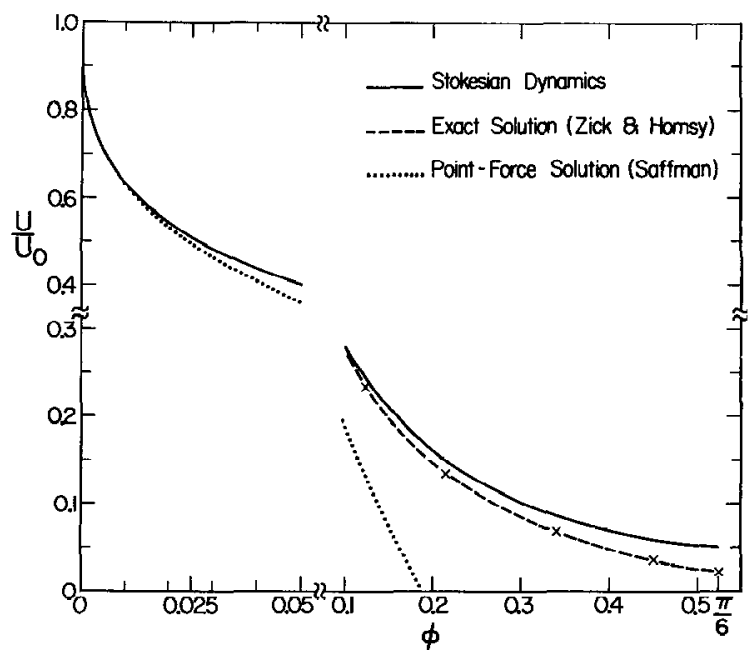

Figure 3 Nondimensional sedimentation velocity of a simple cubic array of spheres as a function of volume fraction $\phi$. The solid curve is the result of the Stokesian-dynamics method, the dashed curve is the exact result of Zick \& Homsy (1982), and the dotted curve is the point-force solution of Saffman (1973). To facilitate comparison at high and low $\phi$, the ordinate and abscissa scales change for $\phi \geq 0.1$. The exact and Stokesian-dynamics results are indistinguishable up to $\phi=0.1$.

summed mobility matrix gives identically the point-force calculation of Saffman. Note, however, that the point-force solution badly underestimates the sedimentation velocity for $\phi>0.1$ and becomes negative for $\phi \approx 0.19$. Including the finite size of the particles, i.e. approximating the force density as constant on the particle surface rather than at its center, is sufficient to remove this aphysical behavior.

Another comparison that demonstrates the importance of the lubrication forces and the fact that they are properly included is the shear viscosity of a simple cubic array of spheres. Nunan \& Keller (1984) have solved this problem following the procedure of Zick \& Homsy (1982); the dilute and close-packed limits were first worked out by Zuzovsky et al. (1983). There are two scalar functions relating the bulk stress to the rate of strain for cubic lattices, i.e. the fourth-order tensor $\mathbf{R}_{\mathrm{SE}}$ in (14a) giving the particle contribution to the bulk stress can be written uniquely in terms of these two scalars. [Note that for a sheared periodic suspension we have $\mathbf{U} \equiv \mathbf{U}^{\infty}$, and thus only the second term contributes in (14a).] Figure 4 shows one of the scalar functions, $\alpha$, as a function of $\phi$. The dashed curve is the "exact" result of Nunan \& Keller (1984), the dotted curve is the result of just using $\left(\mathbf{M}_{\mathrm{ES}}^{*}\right)^{-1}$ (i.e. the far-field Ewald-summed contribu- 


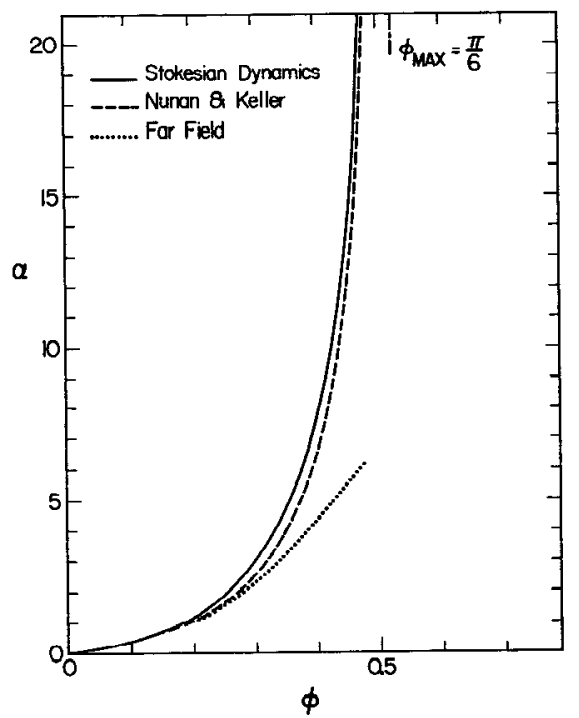

Figure 4 Comparison of the shear-viscosity coefficient $\alpha$ for a simple cubic array of spheres as a function of volume fraction. The solid curve is the Stokesian-dynamics result, the dashed curve is the exact solution of Nunan \& Keller (1984), and the dotted curve is the result of using only the far-field Ewald-summed stresslet-rate of strain coupling $\left(\mathbf{M}_{\mathrm{ES}}^{*}\right)^{-1}$, i.e. lubrication has not been included. The Stokesian-dynamics and the exact results have the same asymptotic form as $\phi \rightarrow \phi_{\max }=\pi / 6$.

tion with no lubrication), and the solid curve is the Stokesian-dynamics calculation with lubrication. Agreement is again seen to be quite good, being exact as $\phi \rightarrow 0$ [specifically, to $O\left(\phi^{2}\right)$ ] and as $\phi \rightarrow \phi_{\max }$, where $\alpha \sim 3 \pi / 16 \varepsilon^{-1}-27 \pi / 80 \ln \varepsilon+\cdots$, and $\varepsilon=1-\left(\phi / \phi_{\max }\right)^{1 / 3}$. Agreement for the other simple cubic lattices for this case and for the sedimentation problem is comparable.

These periodic suspension calculations are static results, with the kinematics dictated by the periodicity, but they are the simplest and the only exact calculations for concentrated suspensions. From these simple examples we see that Stokesian dynamics captures the essential physics of the many-body interactions and the lubrication forces. Clearly, all the qualitative behavior of concentrated flowing supensions is properly modeled, and the quantitative agreement is also expected to be quite good.

The method we have outlined here should also find application in conventional molecular-dynamics simulations of particles with long-range interactions. Indeed, Ewald sums have long been used in the simulation of dipolar fluids, electrolytes, and plasmas. The closest analog in these 


\section{BRADY \& BOSSIS}

molecular systems is the so-called one-component plasma (Levesque et al. 1986 ) in which there are heavy (and hence slowly moving) positive ions immersed in a "sea" of rapidly moving electrons. The smearing out of the negative charge plays the same role as the incompressibility $(\nabla \cdot \mathbf{u}=0)$ in the Stokes-flow problem. The construction of the electrostatic potential in the one-component plasma is analogous to (20) for the fluid velocity $\mathbf{u}$. The analogy stops there, however, because in Stokesian dynamics the velocity field is used directly in the dynamics to move particles, whereas in the plasma case the gradient of the potential gives the force on an ion and Newton's laws of motion are used. The initial kinetic energy of the particles must also be specified in the plasma case (only particle positions are needed in Stokesian dynamics, not initial positions and velocities), and there is a natural parameter measuring the relative importance of electrostatic and thermal energy that determines the plasma behavior. No such parameter is present in Stokesian dynamics.

Similar analogies can be made with the electrolyte case, where the assumption of an equal number of sedimenting and buoyant particles $(\langle\mathbf{F}\rangle=0)$ is equivalent to charge neutrality, and with the dipolar fluid, where there are force-free particles in shear flow (just the $\mathbf{M}_{\mathrm{ES}}$ interactions). However, the analogies are not perfect, primarily because only positions need be given in Stokesian dynamics, whereas positions and velocities are needed in the molecular systems. Although Ewald sums and effective medium calculations (only possible for the conditionally convergent dipolar-fluid case) have long been used in such systems (de Leeuw et al. 1983), no equivalent simple, lucid analysis such as $O^{\prime}$ Brien's seems to have been developed. O'Brien's analysis applies equally well, however, to these electrostatic systems (Bonnecaze 1987).

This concludes our discussion of the Stokesian-dynamics method. The problem formulation for $N$ particles in a volume $V$ and the use of O'Brien's method to construct convergent expressions are exact. The only approximations introduced are the calculation of the hydrodynamic interactions and the use of periodic boundary conditions to simulate an unbounded medium. Both of these approximations are of a computational nature and can be systematically improved by including more moments in the calculation of the hydrodynamic interactions and more particles in the simulation.

\section{APPLICATIONS}

The Stokesian-dynamics simulation method can be applied to a wide variety of problems in the biological, chemical, and physical sciences, as well as to problems of chemical, civil, materials, and mechanical engi- 
neering interest. Here, we illustrate its use in some select examples. The selection of sample problems is largely dictated by the fact that Stokesian dynamics is in its infancy, and thus results are only available for a very restricted class of problems that arose out of our initial interests. Four separate topics are discussed: rheology, diffusion, porous media, and the simulation of systems with physical boundaries. We hope that these few examples will give an indication of the diverse types of information that can be obtained by simulation.

\section{Rheology}

One of the first applications of Stokesian dynamics was a study of the rheology of a concentrated suspension of spheres (Bossis \& Brady 1984, Brady \& Bossis 1985). The model suspension consisted of a monolayer of identical spheres immersed in a simple shear flow; the particles all lie in the $(x, y)$ plane, which is the plane of shear. A monolayer was chosen because the number of degrees of freedom per particle reduces from 11 to 6 , with a large computational savings. At the same time, the physics of the particle interactions within the plane of shear is the same as that in three dimensions, so this model should at least qualitatively describe the behavior of three-dimensional suspensions. Furthermore, experiments have been performed on such monolayer suspensions, as they allow an easy viewing of the suspension microstructure (Bouillot et al. 1982, Blanc et al. 1983, Camoin et al. 1985).

In these simulations generally 25 particles were used, and more recent calculations with 49 particles give statistically the same results. In these early works, the full mobility inversion method outlined in the previous section was not employed; rather, only a pairwise additivity of forces in the resistance matrix was used, i.e. only $\mathscr{R}_{2 \mathrm{~B}}$ in Equation (18). This method captures the lubrication singularities at close particle spacings, but it does a poor job on the many-body interactions. In the monolayer, however, the many-body interactions are less important because the "volume" fraction of particles in a three-dimensional sense is actually zero. There is now an areal fraction $\phi_{\mathrm{A}}=N \pi a^{2} / A$, where $A$ is the area of a periodic cell and $N$ is the number of particles. Also, because of the monolayer, many of the divergences associated with the long-range hydrodynamic interactions disappear, and use of the Ewald summation method is not essential for suspensions in which the average force is zero. Only the viscosity results discussed in this subsection employed the pairwise additivity of forces; all other calculations reported used the full mobility inversion method. Recent simulations using the full inversion method for the viscosity problem resulted in the same qualitative behavior as that discussed below, although quantitative differences were sometimes present. 


\section{6}

BRADY \& BOSSIS

One of the most interesting aspects observed by simulation was the microstructure formed in sheared suspensions and its profound influence on the suspension rheology. In Figure 5 we show the pair-distribution function $g(\mathbf{r})$ obtained by simulation for a monolayer of spheres at an areal fraction $\phi_{\mathrm{A}}=0.4$. Here $g(\mathbf{r})$ measures the probability density of finding a second particle a distance $\mathbf{r}$ from a test particle and is, in general, a function of both $r$ and $\theta$. In Figure 5 a simple $\theta$ average, $\langle g(r)\rangle_{\theta}$, of $g(\mathbf{r})$ is shown for two cases. The solid curve is the pure hydrodynamic case [i.e. $\mathbf{F}^{\mathbf{P}}=0$,

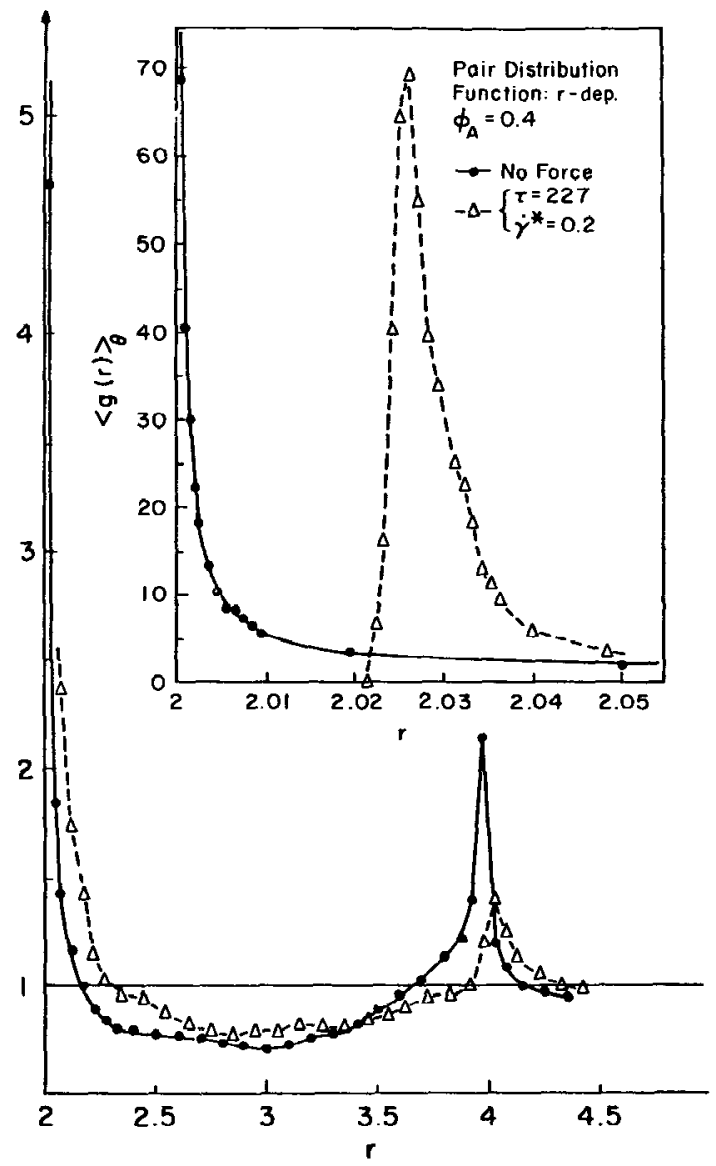

Figure 5 The radial dependence of the pair-distribution function $\langle g(r)\rangle_{\theta}$, which is a simple $\theta$-average, at ${ }^{\top} \phi_{A}=0.4$. The solid curve $(O)$ is the pure hydrodynamic distribution, and the dashed curve $(\triangle)$ is the distribution in the presence of repulsive interparticle forces at $\dot{\gamma}^{*}=0.2$ and $\tau=227$ (from Brady \& Bossis 1985). 
$\mathrm{F}^{\mathrm{B}}=0$ in Equation (1) or $\dot{\gamma}^{*-1}=0, \mathrm{Pe}^{-1}=0$ in Equation (4)] and the dashed curve is a non-Brownian $\left(\mathbf{F}^{\mathrm{B}}=0\right.$ or $\left.\mathrm{Pe}^{-1}=0\right)$ suspension of spheres with a short-range repulsive interparticle force. Specifically, the pairwise-repulsive force between particles $\alpha$ and $\beta$ is along the line of centers and is given by

$$
\mathbf{F}^{\alpha \beta}=\mathbf{F}_{0} \frac{\tau e^{-\tau \xi}}{1-e^{-\tau \xi}},
$$

where $\xi$ is the dimensioniess separation between particle surfaces, $\tau$ is a dimensionless parameter setting the range of the interparticle force and having a value of 227 in these simulations, and $\mathbf{F}_{\mathbf{0}}$ is the force amplitude. The parameter $\dot{\gamma}^{*}=6 \pi \eta a^{2} \dot{\gamma} /\left|\mathbf{F}_{0}\right|$ is the dimensionless shear rate and has a value of 0.2 in Figure 5 . This form of the interparticle force corresponds to charged particles interacting through DLVO-type forces at constant surface charge.

The most important feature to note in Figure 5 is the very sharp firstnearest-neighbor peak in $\langle g(r)\rangle_{\theta}$. With the repulsive force, $\langle g(r)\rangle_{\theta}$ peaks where the shear and interparticle forces balance on the upstream side of the reference particle, i.e. where the convection of the second particle by the shear flow is toward the reference particle. In the pure hydrodynamic limit, $\langle g(r)\rangle_{\theta}$ is actually singular as $r \rightarrow 2$, since particle pairs are "stuck" together by the lubrication forces. The amplitude and sharpness of these peaks are to be contrasted with the pair distribution present in Brownian suspensions or in molecular hard-sphere systems (Figure 9).

The pair-distribution function gives one measure of the structure in suspensions, but it is neither the only nor necessarily the most important one. In fact, we have found that the formation of larger aggregates or clusters is far more important in controlling the macroscopic properties of suspensions. In Figure 6 we illustrate a typical microstructure with a "snapshot" of a sheared monolayer of 49 particles interacting purely hydrodynamically. One should note the formation of a large cluster along the compressive axis of the shear flow $\left(135^{\circ}\right)$ encompassing most of the particles. The large clusters rotate more or less en masse, although they display complex internal dynamics. This is illustrated in the figure, where the lines in each particle indicate the magnitude and direction of the particle's translational velocity relative to the overall bulk shearing motion (which is to the right at the top and to the left at the bottom of the figure). When the cluster is aligned along the compressive and extensional axes, the instantaneous suspension viscosity is largest; when the orientation is vertical, the intensity of the internal motion increases and the viscosity is smallest. In the simulations the total stress tensor $\langle\boldsymbol{\Sigma}\rangle$ from (13) is calculated, and the viscosity is simply the ratio of $\left\langle\Sigma_{x y}\right\rangle$ to the rate of strain 


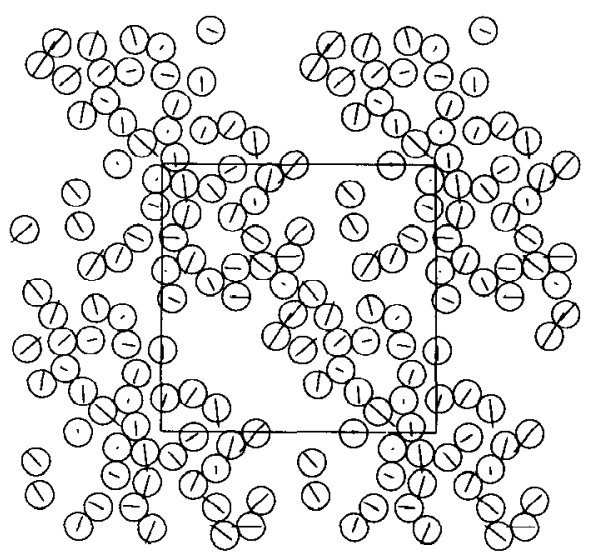

Figure 6 A typical particle configuration of a sheared monolayer of purely hydrodynamically interacting spheres at an areal fraction $\phi_{A}=0.4$. The bulk motion is to the right at top and to the left at bottom. Note the formation of a large cluster along the compressive axis $\left(135^{\circ}\right)$ of the shear flow. The lines in the particles give the magnitude and direction of the particle translational motion relative to the average shear flow. The relative instantaneous suspension viscosity is highest when the clusters are oriented along the compressive and extensional axes, and lowest when the orientation is vertical (from Bossis \& Brady 1987).

$E_{x y}^{\infty}$. For this purely hydrodynamic suspension, the only contribution the particles make to the bulk stress is through $\left\langle\mathbf{S}^{\mathrm{H}}\right\rangle$.

Our studies have shown that it is the cluster formation that is responsible for the increase in suspension viscosity with increasing volume (or areal) fraction. The clusters are able to span large regions of the flow and effectively transmit stress over considerable distances. In a crude sense, the clusters can be viewed as rigid rods, held together by the lubrication forces and the overall connectivity of the structure. As discussed in the introduction, the stress in a rod grows roughly as the length cubed; thus, this explains the large viscosities. With increasing volume fraction the average cluster size grows, and our simulations suggest a percolation-like phenomenon with a singular viscosity as $\phi$ approaches some $\phi_{\max }$.

Another way to view the effect of the clusters is through the average relative radial velocity of particle pairs in suspension. Because of the lubrication singularities, the dominant contribution to the suspension viscosity comes from the relative radial motion of the particles. This is illustrated in Figure 7, where the ratio of the actual relative radial velocity of two spheres in suspension to that which they would have if there were only two particles, $V_{\mathrm{r}}$, is plotted as a function of $r$. For two spheres alone in the fluid, the relative radial velocity $\Delta U_{\mathrm{r}}^{2 \mathrm{~B}}$ approaches zero as the particles come near contact-specifically, $\Delta U_{\mathrm{r}}^{2 \mathrm{~B}} \sim-4.077 \xi$ as $\xi \rightarrow 0$, 


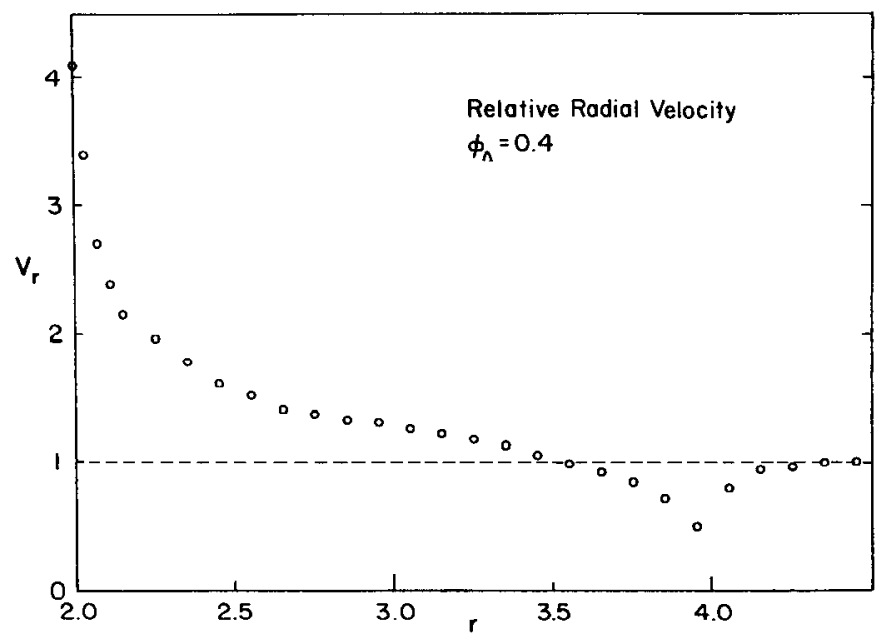

Figure 7 The relative radial velocity $V_{\mathrm{r}}$ of two spheres as a function of separation $r$ at an areal fraction $\phi_{\mathrm{A}}=0.4 . V_{\mathrm{r}}$ is the ratio of the actual relative velocity to that of two spheres alone in the fluid (from Brady \& Bossis 1985).

where $\xi=r-2 . V_{\mathrm{r}}$ follows closely the pair-distribution function and approaches unity for large $r$ as the motion of the two particles becomes uncorrelated. It is as if two nearly touching particles in suspension find themselves in an "effective" shear rate, which at $\phi_{\mathrm{A}}=0.4$ is approximately four times greater than the actual shear rate. The stresses transmitted by the large clusters squeeze particle pairs together (and pull them apart) much more rapidly than for two isolated particles. The effective viscosity scales directly with this effective shear rate.

As a final illustration of the role of aggregate formation, in Figure 8 the relative viscosity of the suspension, $\eta_{\mathrm{r}}$, defined as the ratio of the suspension viscosity to the pure fluid viscosity, with interparticle forces is plotted as a function of the shear rate $\dot{\gamma}^{*}$. What is interesting to note here is that even at a shear rate of $\dot{\gamma}^{*}=10^{4}$, i.e. dividing $\mathbf{F}^{\mathrm{P}}$ in the evolution equation (4) by $10^{4}$, the viscosity is still much smaller than it is in the absence of any interparticle force. At this areal fraction the pure hydrodynamic viscosity is approximately 4. If one examines the pair-distribution function, it would be difficult to distinguish it from the pure hydrodynamic case shown in Figure 5. The explanation for the smaller viscosities with essentially the same $g(r)$ is that the repulsive force, although quite weak, is still very effective at disrupting the large clusters that are present in the pure hydrodynamic case. On the downstream side of a particle, both the shear and the repulsive forces separate particle pairs, and this extra separation is 


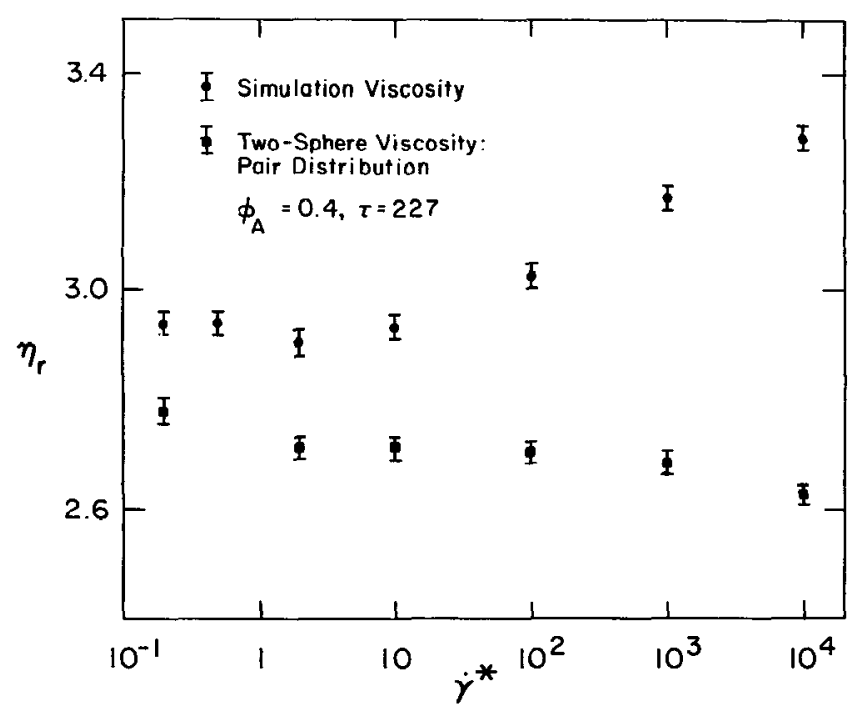

Figure 8 The shear-rate dependence of the suspension viscosity for particles interacting through short-range repulsive forces at an areal fraction $\phi_{\mathrm{A}}=0.4$. The simulations show a shear-thickening behavior due to the increased cluster formation as $\dot{\gamma}^{*} \rightarrow \infty$. The two-sphere viscosities show a shear-thinning bchavior because they contain no information on cluster behavior (from Brady \& Bossis 1985).

sufficient to break the connectivity of the largest clusters. Indeed, the shearthickening behavior this suspension displays results from the formation of larger and larger clusters as the shear rate increases, despite the fact that the interparticle force contribution to the bulk stress, $\left\langle\mathbf{S}^{\mathbf{P}}\right\rangle$ in Equation (13), is proportional to $1 / \hat{\gamma}^{*}$ and is shear-thinning.

Thus the importance of cluster formation, which results from the lubrication forces and excluded volume, cannot be underestimated in understanding suspension behavior. These notions are generally absent from most theoretical treatments of suspension viscosity. The behavior we have observed cannot be obtained by any pairwise additivity of velocities or mobility interactions, as is customary in theoretical studies. Indeed, the lower set of data in Figure 8 shows the results one would get by a pairwise additivity of mobility interactions using the correct pair-distribution function. Not only is there quantitative disagreement, but the qualitative behavior-shear thinning-is just the opposite of what actually occurs.

\section{Diffusion}

Unlike the previous subsection, which focused on the purely hydrodynamic limit, here we discuss the other extreme of Brownian motion-dominated 
behavior. Simulations were again performed for a monolayer of 25 identical spheres at an areal fraction $\phi_{\mathrm{A}}=0.453$ for several values of the Péclet number (Bossis \& Brady 1987). There were no interparticle forces.

In Figure 9 we show a comparison of the radial distribution function, $g(r)$, obtained by simulation for the pure Brownian case $(\mathrm{Pe}=0)$ with the distribution function for a system of hard disks in vacuum obtained by a Monte Carlo method (Chae et al. 1969). That the two distributions should be the same is most easily seen from the Fokker-Planck equation [(5) with (6)]. At equilibrium, the solution for $P(\mathbf{x})$ is just the Boltzmann distribution $\exp [-V(\mathbf{x}) / k T]$, where $V(\mathbf{x})$ is the hard-sphere (or hard-disk) interparticle potential. Although it is true that the Boltzmann distribution must be obtained regardless of what hydrodynamics is used, the process of obtaining $g(r)$ through simulation, where there are no interactions other than hydrodynamic ones $\left(\mathbf{F}^{\mathrm{P}}=0\right)$, is a nontrivial task. It may at first seem surprising that through simulation the correct $g(r)$ is obtained with the interparticle force set equal to zero $\left(\mathbf{F}^{\mathrm{P}}=0\right)$, while the Fokker-Planck equation requires that we write $\mathbf{F}^{\mathrm{P}}=-\nabla V(\mathbf{x})$, the gradient of the hardsphere potential. There is no conflict here because the hard-sphere potential results in repulsive interparticle forces that are delta functions located on the particle surfaces, $\delta(\xi)$, where $\xi$ is the dimensionless surface-surface separation (i.e. the force is only nonzero when particles touch). In the evolution equation (4) these forces are multiplied by $\mathbf{R}_{\mathrm{FU}}^{-1}$, and the relative mobility of two particles vanishes when they touch. Thus, the velocity a particle receives from the hard-sphere repulsion is proportional to $\xi \delta(\xi)$,

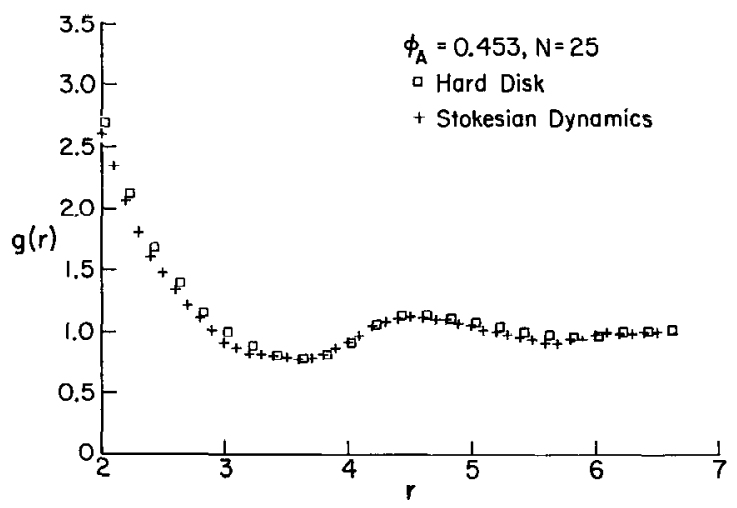

Figure 9 The radial dependence of the pair-distribution function for a purely Brownian suspension at an areal fraction $\phi_{\mathrm{A}}=0.453$ determined by simulation $(+)$ is compared with that of a hard-disk fluid ( $\square$ ) determined by Chae et al. (1969) using a Monte Carlo method (from Bossis \& Brady 1987). 
which is zero; with hydrodynamic interactions, the hard-sphere potential has no dynamical significance. In simulation it is the balance between $\nabla \cdot \mathbf{R}_{\mathrm{FU}}^{-1}$ and the random step $\mathbf{X}$ in the evolution equation (4) that results in the correct physics. Only through a proper treatment of the hydrodynamic interactions - both many-body and lubrication-is the correct $g(r)$ produced.

Stokesian dynamics allows the determination of both the short- and long-time self-diffusion coefficients, defined by (10) and (11), respectively. Both of these are accessible by dynamic light scattering. In Figure 10 we show a plot of the mean-square displacement $\left\langle y^{2}\right\rangle$ vs. time for a monolayer suspension at $\phi_{\Lambda}=0.453$. The transition from the short-time behavior, where $D_{0}^{\mathrm{s}} \approx 0.75$ to the long-time behavior, where $D_{\text {so }}^{\mathrm{s}} \approx 0.48$, can clearly be seen for $\mathrm{Pe}=0$. Once a particle has diffused a distance of order its size, it must exchange places with its neighbors and its motion is slowed down. The changes in slope evident at $\left\langle y^{2}\right\rangle \approx 2.5$ and 6 are caused by motion past the first- and second-nearest-neighbor shells. Also shown in Figure 10 is the behavior for $\mathrm{Pe}=1$ and 10 . The $\mathrm{Pe}=1$ curve follows closely the pure Brownian behavior, indicating that as far as self diffusion is concerned a Péclet number of unity is only a small departure from $P e=0$.

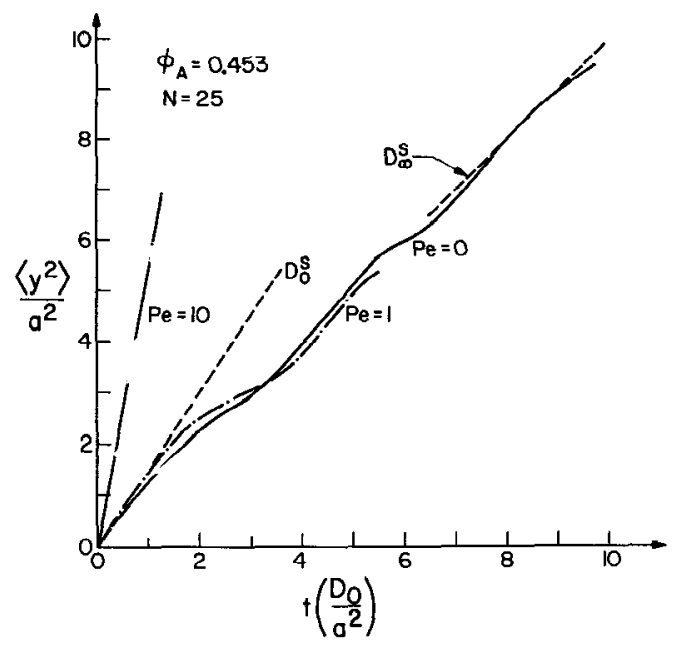

Figure 10 The mean-square displacement $\left\langle y^{2}\right\rangle$ as a function of time $t$ for a monolayer susp:ension at an areal fraction $\phi_{\mathrm{A}}=0.453$ for $\mathrm{Pe}=0,1$, and $10 . D_{0}^{\mathrm{s}}$ and $D_{00}^{\mathrm{s}}$, shown as dashed lines, are the short- and long-time asymptotes for $\mathrm{Pe}=0$, respectively. Note the changes in slope at $\left\langle y^{2}\right\rangle \approx 2.5$ and 6 as the diffusing particle passes its first- and secondnearest-neighbor shells. The behavior for $\mathrm{Pe}=1$ is quantitatively similar to that at $\mathrm{Pe}=0$, whereas for $\mathrm{Pc}=10$ the time scale and amplitude of the motion are quite different, as this is well into the hydrodynamically dominated regime (from Bossis \& Brady 1987). 
The behavior for $\mathrm{Pe}=10$ is, however, quite different. The mean-square displacement rises rapidly because time is being measured in diffusive time units $a^{2} / D_{0}$. At Péclet numbers greater than unity, the shear rate $\dot{\gamma}^{-1}$ should be used as the time scale. Nonetheless, one sees that the long-time self diffusivity is much larger at $\mathrm{Pe}=10, D_{\infty}^{\mathrm{s}} \approx 2.7$ than at $\mathrm{Pe}=0$. As the Péclet number increases, $D_{\infty}^{\mathrm{s}}$ continues to grow, and one anticipates that ultimately $D_{\infty}^{\mathrm{s}}$ should scale as $\mathrm{Pe}$. In the pure hydrodynamic limit $\mathrm{Pe} \rightarrow \infty$ (or $\mathrm{Pe}^{-1}=0$ ), the long-time self-diffusion coefficient should scale with $\dot{\gamma} a^{2}$. Recently, Leighton \& Acrivos (1987) have measured experimentally this shear-induced or hydrodynamic diffusion coefficient, showing that at $\mathrm{Pe}^{-1}=0$ the mean-square displacement of a particle is diffusive and the self-diffusion coefficient does indeed scale as $\dot{\gamma} a^{2}$. Our simulation studies have verified this scaling, and our pure hydrodynamic diffusion coefficients are in good agreement with the experimental values.

As a final illustration of diffusional behavior in suspensions, in Figure 11 we show the relative mobility or diffusion $D_{r}$ of two particles in an isotropic suspension. The relative diffusion coefficient only depends on the vector separation of two particles in an isotropic suspension and can be written in dimensionless form as $\mathbf{D}_{\mathbf{r}}(\mathbf{r})=[G(r) \hat{\mathbf{r}} \mathbf{\hat { r }}+H(r)(\mathbf{I}-\hat{\mathbf{r}} \hat{\mathbf{r}})]$, where $\hat{\mathbf{r}}$ is a unit vector along the line joining the particle centers. The radial component $G(r)$ is shown in the figure, where the solid curve is the result for two spheres alone in the fluid (Batchelor 1976b), and the +'s are for two spheres in a monolayer suspension at $\phi_{\mathrm{A}}=0.453$ and $\mathrm{Pe}=0$. In contrast to the relative radial velocity in a high-Péclet-number sheared suspension shown in Figure 7, the relative radial diffusion of two particles near contact

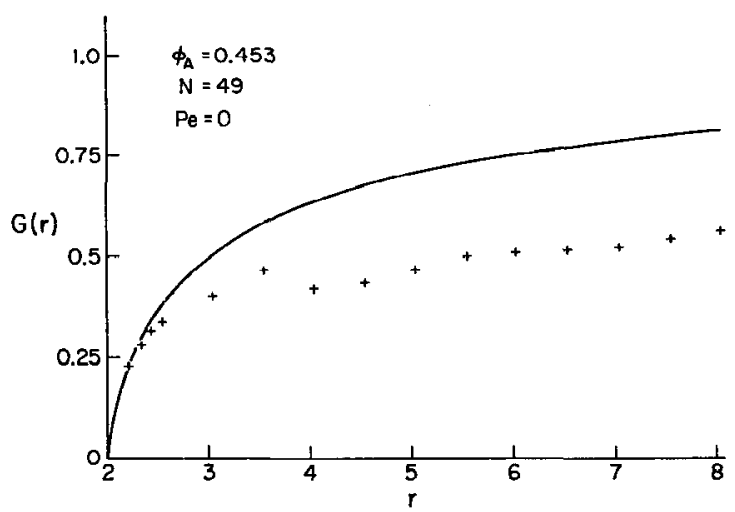

Figure 11 The relative radial mobility or diffusion of two spheres in a Brownian suspension $(\mathrm{Pe}=0)$ at $\phi_{\mathrm{A}}=0.453$. The solid curve is the behavior of two spheres alone in the fluid, and the +'s give the behavior in suspension. The depression evident at $r=4$ is due to the passage of the second-nearest-neighbor shell. 
is the same as if the particles were alone (the tangential motion is not the same, however); at large separations, however, each particle moves independently with a self-diffusion coefficient that is equal to its short-time value. Concepts such as this of an effective two-sphere relative-diffusion coefficient or an effective shear rate may be useful in constructing analytical theories of concentrated suspensions. It should be appreciated, however, that a simple change of scale or amplitude is not sufficient, because the change in scale is not uniform for all separations $r$, nor is it necessarily the same in the radial and tangential directions.

\section{Porous Media}

The previous two problems were concerned with dynamic simulation of suspensions in which the particles were free to move in response to hydrodynamic or Brownian forces. Here we examine a completely different problem - that of the flow in a porous medium where the particles are prescribed to remain fixed. One quantity of interest is the permeability of the porous medium, as defined in Equation (8). In periodic arrays the sedimentation velocity and permeability are the same apart from a multiplicative factor of $\phi$, and the simple cubic sedimentation velocities shown in Figure 3 also give the permeability.

The permeability is only one macroscopic measure of a porous medium, and it is much more interesting to take a more detailed look. The interest comes from the fact that in a porous medium the long-range nature of the hydrodynamic interactions results in a screening of interactions. The velocity disturbance caused by a point force in a dilute, random porous medium should satisfy the Brinkman (1947) equation

$$
\begin{aligned}
& \eta \nabla^{2} \mathbf{u}-\nabla p-\eta \alpha^{2} \mathbf{u}=0, \\
& \nabla \cdot \mathbf{u}=0,
\end{aligned}
$$

where $\mathbf{u}$ and $p$ are the suspension average velocity and pressure, respectively, $\eta$ is the fluid viscosity, and $\alpha^{-2}$ is the permeability.

On small length scales, the pressure gradient balances the Laplacian of the velocity, and thus the flow is essentially viscous. On large length scales, where the velocity is slowly varying, the pressure gradient balances the average velocity as in Darcy's law. The characteristic length that distinguishes these two regions is the Brinkman screening length given by the square root of the permeability $\alpha^{-1}$. In the dilute limit, we have $\alpha^{-1}=\left(2^{1 / 2} / 3\right) a \phi^{-1 / 2}$, where $a$ is the characteristic particle size and $\phi$ is the volume fraction of solids. In a viscous fluid, the velocity disturbance due to a point force decays as $1 / r$, whereas in a dilute porous medium at distances larger than $\alpha^{-1}$, the disturbance is screened and decays faster, as 
$1 /\left(\alpha^{2} r^{3}\right)$. Although Brinkman's derivation of (22) was heuristic, subsequent investigators have formally established its validity asymptotically at low $\phi$ (Tam 1969, Childress 1972, Saffman 1973, Howells 1974, Hinch 1977).

We have investigated the validity of the Brinkman equation as a model for interactions in a porous medium with Stokesian dynamics (Durlofsky \& Brady 1987). This is both a check on the correctness of the Brinkman equation (for there are no experimental results for the flow in dilute, random porous media) and on our Stokesian-dynamics method (for only by properly treating the many-body interactions will the correct behavior be produced). This also demonstrates the utility of Stokesian dynamics for problems other than simulation and shows that very diverse hydrodynamic problems can all be treated within the same framework.

The response to a point force in a porous medium is the same as the interaction between two particles in the resistance matrix. Recall that an $\alpha \beta$ element of the resistance matrix $\mathbf{R}_{\mathrm{FU}}^{\alpha \beta}$ [cf. Equation (2)] gives the force on particle $\alpha$ due to the velocity of particle $\beta$, with all other particles held fixed. To determine the nature of the interactions, we generated random samples of 125 particles in three dimensions, applied a force to one particle of a pair, and measured the velocity response of the other as a function of separation. In these dilute stationary systems, the full moments expansion discussed in the simulation method is not necessary, and only point forces with Ewald sums were used. In a dilute porous medium this should generate the Green function to Brinkman's equation, which is given by

$$
\mathscr{J}=\frac{4}{3} f(r) \mathbf{I}+\frac{4}{3} g(r) \hat{\mathbf{r}}
$$

where $\hat{\mathbf{r}}$ is a unit vector in the radial direction (along the line connecting the two particles in simulation), and the scalar functions $f(r)$ and $g(r)$ are given by

$$
\begin{aligned}
& f(r)=\frac{3}{2 \alpha^{2} r^{3}}\left[\left(1+\alpha r+\alpha^{2} r^{2}\right) e^{-\alpha r}-1\right], \\
& g(r)=\frac{9}{2 \alpha^{2} r^{3}}\left[1-\left(1+\alpha r+\frac{1}{3} \alpha^{2} r^{2}\right) e^{-\alpha r}\right] .
\end{aligned}
$$

In Figure 12 we show a comparison of the scalar functions $f$ and $g$ with the results obtained by Stokesian dynamics at a volume fraction $\phi=0.002$. The dashed curves shown are the corresponding scalar functions for the Stokes-flow Green function, which are the $\alpha \rightarrow 0$ limits of (24) and are both $3 /(4 r)$. We see clearly that particle interactions are screened in a porous medium and are well described by the Brinkman equation for dilute 

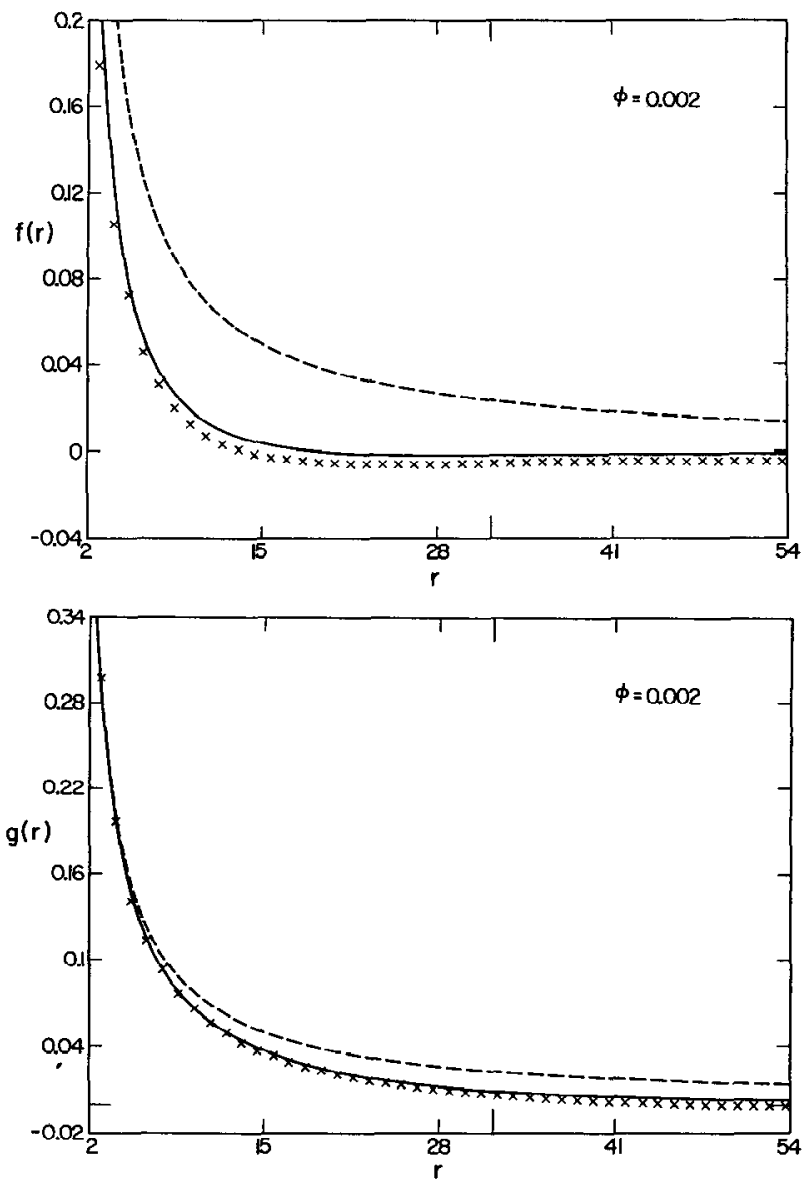

Figure 12 Comparison of the theoretical Brinkman Green function (solid curves) in Equations (21)-(22) for (top) $f(r)$ and (bottom) $g(r)$ with the results from simulation (X) at $\phi=0.002$ with 125 point-force particles. The dashed curves are the corresponding Stokesflow Green functions. The slight offsets between the simulation and Brinkman results are a finite-size effect and scale as $1 / N$, where $N$ is the number of particles in simulation (from Durlofsky \& Brady 1987).

systems. The slight offset of the Stokesian-dynamics results from the Brinkman solution is due to the finite number of particles used and scales as $1 / N$. Qualitatively incorrect behavior results if Ewald summations are not used. The large tick mark on the abscissa in the figures denotes the halflength of the periodic cell. In order to model a random medium, the Brinkman screening length must fit within the box half-length, giving the requirement that $\left(3 / 2^{1 / 2}\right)(\pi / 6)^{1 / 3} N^{1 / 3} \phi^{1 / 6}>1$, which is satisfied here. 
These results validate both the Brinkman equation as a description of interactions in a dilute porous medium and the ability of Stokesian dynamics to accurately model porous media. As might be expected, as the volume fraction increases $(\phi>0.2)$, the Brinkman equation no longer provides a quantitative description of particle interactions, although the screening is, of course, still present. This opens a question as to the use of the Brinkman equation at high $\phi$, despite the fact that permeabilities obtained from solving a two-body problem in a Brinkman medium are in good agreement with experiment (Kim \& Russel 1985). The permeability is rather insensitive to the details of the velocity field, with the largest contribution coming from the pressure drop across a particle. This insensitivity illustrates that although a model may describe one overall macroscopic property, it is not necessarily a complete or correct model.

\section{Bounded Suspensions}

The previously discussed examples all dealt with unbounded suspensions. In this last application of Stokesian dynamics, we examine the influence of physical boundaries on suspension behavior. Boundary effects can be important for two quite different reasons. First, because of the long-range nature of the hydrodynamic interactions, even very distant boundaries can significantly influence a particle's motion, especially if these boundaries are in the form of infinite planes. Second, because of the tendency of sheared suspensions to form clusters, at large enough volume fractions percolating clusters that span the gap between moving plates may form, resulting in a boundary-dominated flow even though the ratio of the gap width to particle size is very large and one would normally expect that the boundaries would have little effect on the suspension behavior. The proper criterion for neglecting the effect of the boundaries is, of course, that the ratio of the gap width to cluster size be large, not the gap width to particle size.

A simulation method for bounded flows can be developed along the same lines as that for unbounded flows outlined in the previous section. This has been done for the case of a suspension placed between two infinite parallel planes, which can be set in motion relative to one another to create a deforming motion (Durlofsky 1986). We again start from the integral form of the solution to the Stokes equations and generate a grand mobility matrix analogous to (17). The walls are broken up into a near region (which is discretized into patches that interact with each other and all the suspended particles in a mobility matrix) and a far region (whose average integrated effect on the motion of the particles and wall patches is calculated analytically). The effects of the far region are in many ways analogous to the "back-flow" integrals arising in the unbounded case, for they 
now depend on the average force density on the walls and their translational velocities. This mobility formulation is then inverted, and the short-range lubrication forces, including those between particles and walls, are added in as before. The result is an evolution equation for particle positions very similar to Equation (4), except that in place of the hydrodynamic forces from the impressed shear rate, $\mathbf{R}_{\mathrm{FE}}: \mathbf{E}^{\infty}$, there now appears a term proportional to the relative velocity of the two parallel walls. The relative motion of the walls generates the flow, just as is done experimentally in a shear cell. In this formulation either the total force exerted on the planes can be specified and their resultant velocities (as well as the motion of the particles) found, or else the velocities can be specified and the force required to generate the motion determined. The latter again corresponds to the force measured experimentally in a Couette device. Note also that both the tangential and normal forces exerted on the walls will be calculated, and thus normal-stress differences will be determined.

Using this simulation method we studied the shear viscosity of a monolayer of identical spheres, with the plane of the monolayer oriented perpendicular to the walls. Figure 13 shows a time trace of the shear viscosity, defined as the force per unit area required to move a wall (which

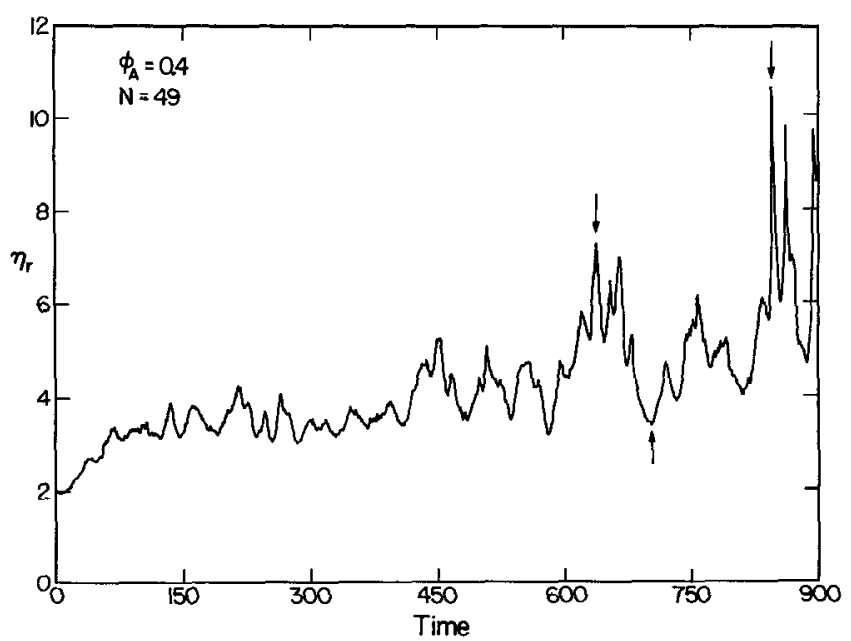

Figure 13 Time trace of the suspension viscosity $\eta_{\mathrm{r}}$ of a monolayer of 49 neutrally buoyant spheres at an areal fraction $\phi_{\mathrm{A}}=0.4$ being sheared between two parallel planes. The viscosity is defined as the force required to move one plate divided by the shear rate $\dot{\gamma}=\Delta U / H$, where $\Delta U$ is the relative velocity of the planes and $H$ is their separation. Time has been nondimensionalized by the particle shear rate $\Delta U / a$, and the ratio of the particle size to gap width is $a / H=0.051$. The large fluctuations in viscosity are the result of clusters that span from one plane to the other (see Figure 14). 
is found from simulation) divided by shear rate $\dot{\gamma}=\Delta U / H$, where $\Delta U$ is the relative tangential velocity of the walls and $H$ is their separation. The trace is for a suspension of 49 neutrally buoyant particles at an areal fraction $\phi_{\mathrm{A}}=0.4$. In the figure one should note the gradual evolution of the suspension viscosity with time and the increasing amplitude of the fluctuations. The particles are initially well dispersed and begin to form large, spanning clusters after a time of 500 . Here the time is expressed in units of the particle shear rate $\Delta U / a$, with $a / H=0.051$, and the suspension has been replicated periodically in the direction of flow with a periodic box length equal to the spacing between the walls.

The very large fluctuations seen for times greater than 600 result from clusters that span from one wall to the other and transmit very large stresses. These clusters dynamically form and break, giving rise to the fluctuations. This sequence is made evident in Figure 14, where snapshots of particle configurations at times corresponding to the arrows in Figure 13 are shown. The sequence shows a spanning cluster with a large viscosity (top), followed by a low-viscosity point where there are no spanning clusters (middle), followed again by a very large-viscosity point and a spanning cluster (bottom). The correlation between viscosity fluctuations and spanning clusters is direct: They always occur together. Note also the percolative nature of the clusters-if one displaces by a very small amount only one sphere in the spanning cluster in Figure 14 (top), the lubrication connectivity will be destroyed and the viscosity will drop by a factor of 4 or more.

These large fluctuations were only observed when the areal fraction exceeded approximately 0.35 . Below this value the fluctuations were of much smaller amplitude, and spanning clusters were not observed. Qualitatively the same behavior occurred when 25 particles were used in place of 49. The fluctuations appeared earlier in time, but only above an areal fraction near 0.35 . These results strongly suggest a percolation-like phenomenon with a critical areal fraction of 0.35 , but one must exercise caution in drawing this conclusion because of the limited time interval simulated and the small simulation sizes. Indeed, it can be seen in Figure 13 that the suspension structure is still evolving in time even after a time of 900 , corresponding to a total strain of $5 H$.

At still higher values of $\phi_{\mathrm{A}}$, we noticed the onset of "plug-flow" behavior in which the particles were all bunched together in a single cluster that translated more or less as a single entity. This is illustrated in Figure 15, where we have plotted the particle average velocity in the flow direction, i.e. parallel to the planes, as a function of the transverse coordinate $y$ for a suspension of 25 neutrally buoyant particles at $\phi_{\mathrm{A}}=0.6$. The dashed line joining the corners in the figure is the velocity profile that a pure fluid 

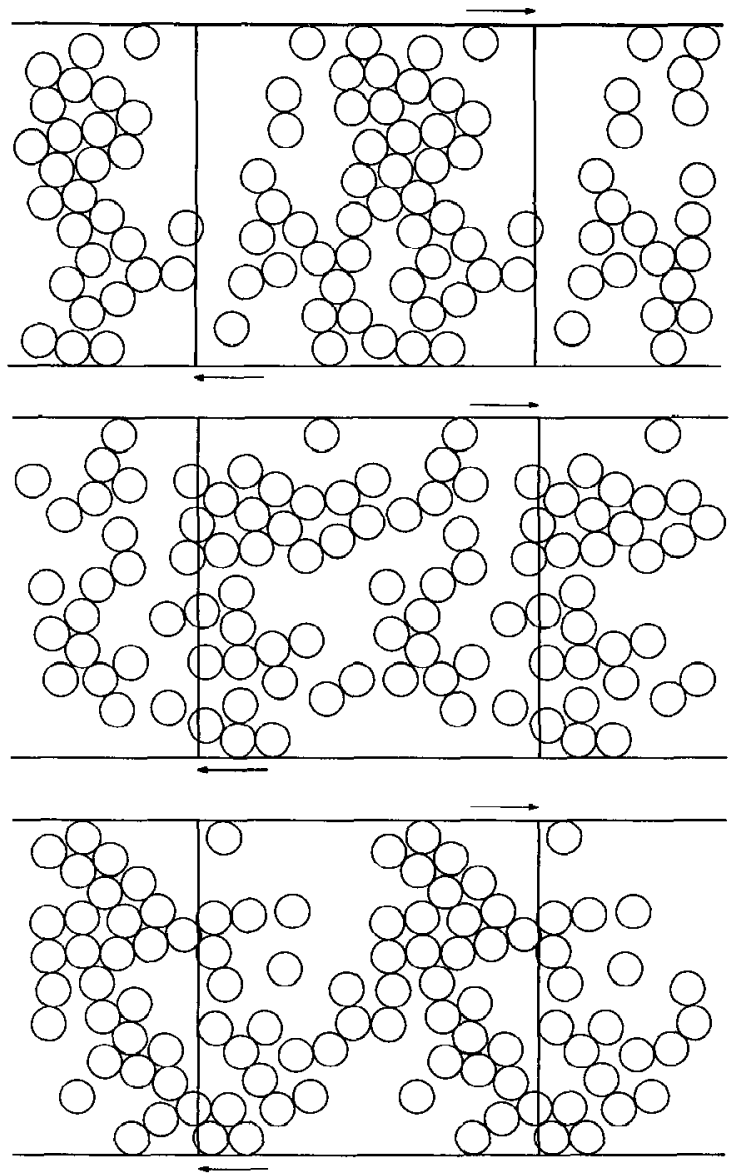

Figure 14 Snapshots of instantaneous particle configurations for the sheared suspension of Figure 13. The sequence (from top to bottom) corresponds in time to that indicated by the arrows in Figure 13. These arrows correspond to the maxima and minima of the viscosity fluctuations. Both the top and bottom frames show the presence of a spanning cluster-a connected path from one wall to the other-and give rise to large viscosities. In the middle frame, no spanning cluster is present and the viscosity is relatively low.

or a dilute suspension would have. The solid curve is a time average of the particle velocities and shows the particles moving as a plug at an average velocity of roughly $1 / 2$, with regions of rapid shear adjacent to the walls. Plug-flow-like behavior has been observed experimentally by Karnis et al. (1966) in concentrated suspensions in both pressure-driven flow in tubes and in shear flow in a cylindrical Couette device. The velocity profiles sketched by Karnis et al. agree qualitatively with those of Figure 15. 


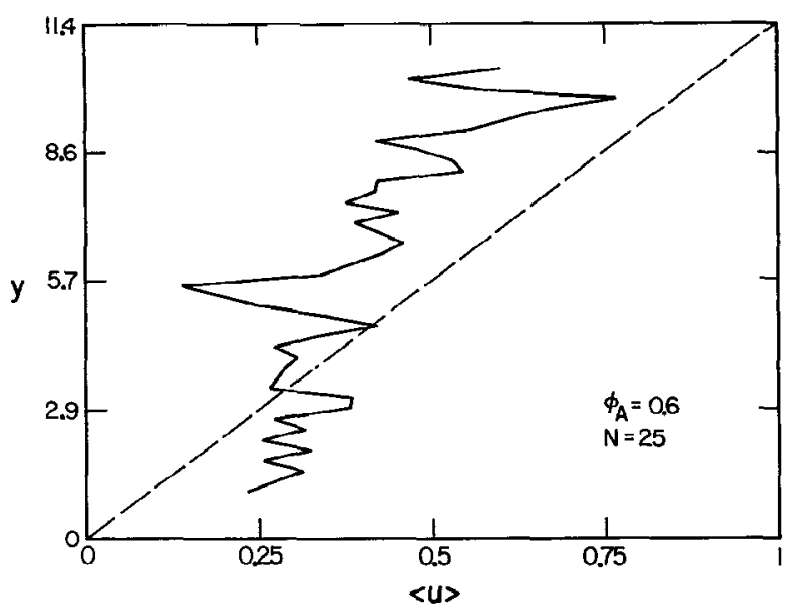

Figure 15 Plot of the particle average veiocity in the flow direction, $\langle u\rangle$, versus the transverse coordinate $y$ for a bounded, sheared suspension of 25 neutrally buoyant spheres at a high areal fraction of $\phi_{\mathrm{A}}=0.6$. The dashed line is the velocity profile a Newtonian fluid would have. Notice the formation of a "plug-flow" region, where all the particles are clustered together and move at roughly half the wall speed, and two regions of rapid shear adjacent to the walls (from Durlofsky 1986).

This last application once again serves to illustrate the profound importance cluster formation has on suspension properties. In concentrated suspensions it seems to be the most important aspect controlling suspension behavior.

\section{CONCLUSIONS}

We hope that the discussion in the previous two sections has demonstrated the accuracy and utility of Stokesian dynamics. A large variety of scientific and engineering problems can now be studied through dynamic simulation, an approach that was nonexistent only a few years ago. By way of conclusion we would like to point out some other general areas, as well as specific problems, for which Stokesian dynamics may prove useful and to indicate those aspects of the simulation method that could be (need to be) improved.

We have implicitly assumed that the reader was well aware of the need and value of simulation in a general sense, although this may not be the case. One of the major advantages of simulation is the ability to control a single parameter and thus isolate its specific effects. This is often a very difficult task to accomplish experimentally. Simulation also provides a 


\section{BRADY \& BOSSIS}

rigorous testing ground for theories. Over the years many theories have been advanced to predict suspension viscosities or sedimentation rates, and the merits of one theory over another are not always obvious, particularly when they all fit the existing experimental data. Theories can now be scrutinized in detail by comparison with simulation, and their merits and limitations clarified. Simulation can also guide the development of new theories (and this has already occurred for us) when phenomena observed in simulation cannot be predicted by any current approach. Finally, and most importantly, simulation can give one new insights, often of simply a qualitative nature, into a system's behavior. The profound role played by particle clustering was not widely appreciated before our simulation studies. It now appears to be the most important feature in concentrated suspensions.

To date, the problems to which Stokesian dynamics has been applied are few in number and represent just the beginning. The general method we have developed is not limited to spherical particles of identical size. Extensions to a distribution of particle sizes or to rod- or disk-shaped particles is straightforward and has already begun (J. D. Sherwood, personal communication). Much more complex objects such as aggregated flocs (Chen et al. 1984, Meakin et al. 1985, Meakin \& Deutch 1987, Adler 1987) or particles with internal degrees of freedom, as in micromechanical models of polymers (Dotson 1983, Saab \& Dotson 1987, Fixman 1987), can also be studied. Simulations can either be of a single complex entity such as a deforming polymer molecule (and one need no longer use approximate or preaveraged hydrodynamics), where the focus is on the internal dynamics, or of a suspension of such objects, where the contribution of the internal degrees of freedom to the macroscopic behavior is sought. Furthermore, the incorporation of physical boundaries into the simulation method can be extended to pressure-driven flows in conduits and can be used to investigate the form of the boundary conditions needed for use in averaged-equation approaches to suspensions, a problematic and often neglected area. Indeed, Stokesian dynamics can be used directly in developing and testing constitutive equations for average suspension behavior.

There is also a large class of problems requiring hydrodynamic interactions for which general formulas exist for computing desired equilibrium or transport properties and a full dynamic simulation is not necessary. For example, in the transport of particulates through porous media the problem can be reduced to solving a convection-diffusion equation followed by an integration (Brenner 1980a,b, 1982, Adler \& Brenner 1982, Koch \& Brady 1985, 1987), and Stokesian dynamics can be used to gen- 
erate the required hydrodynamic data. The method we have developed to calculate the hydrodynamic interactions - a many-body far-field multipole expansion combined with a two-body treatment of the near-field physicsis also applicable to other scalar and vector transport processes. Specifically, conductance-type problems (heat, mass, electric current, electric displacement, magnetic susceptibility) and elasticity problems (bulk and shear moduli) have the same combination of far-and near-field interactions and can be treated in an identical manner (Bonnecaze 1987). The approach may also be adaptable to wave propagation in heterogeneous media.

While the Stokesian-dynamics method is accurate and efficient, it is still computationally intensive. Ideally, determining the interactions among $N$ particles would require $O\left(N^{2}\right)$ operations. Filling the mobility or resistance matrices, as well as computing the Ewald sums for infinite suspensions, also requires $O\left(N^{2}\right)$ operations. However, the many-body interactions obtained by inverting the mobility matrix and the inclusion of lubrication forces in the resistance matrix both require $O\left(N^{3}\right)$ operations and limit the size of systems that can be studied. Thus, an area of activity that can have substantial impact is to devise a computational scheme that is more efficient than the one we have presented. The many-body aspect may be particularly hard to overcome because, at the level of stresslets and the mean-field quadrupole, we are just at the limit of conditional convergence of interactions in suspensions, and many macroscopic properties (e.g. rheology) require knowledge of particle stresslets. Note that the procedure developed in stellar dynamics that reduces the $O\left(N^{2}\right)$ problem to an $O(N \ln N)$ problem by discretizing space and using fast Fourier transforms [see Goodman \& Hut (1985) and Fogelson (1986) for an application to Stokes flow] is not readily adaptable to the hydrodynamic problem, where the force density (equivalent of mass density) is not given but rather is distributed over the particle surface and must be found from the overall total force balance and the requirement that the particle move as a rigid object.

Many researchers who have approached the suspension problem from the Brownian-motion-dominated regime have ignored the $O\left(N^{3}\right)$ nearfield lubrication interactions with the hope that there would be few close particles in suspension, and thus an $O\left(N^{2}\right)$ pairwise-additive mobility interaction would suffice. On the surface this appears to be a substantial computational savings, but further examination reveals that the Brownian displacements in the evolution equation (4) require taking the square root of the mobility matrix, which is in itself an $O\left(N^{3}\right)$ operation. Thus, it would seem that for almost all cases of interest, the hydrodynamic problem is inherently a costly $O\left(N^{3}\right)$ one.

One possible approach for overcoming the $O\left(N^{3}\right)$ problem, however, is 


\section{BRADY \& BOSSIS}

in the simulation of infinite suspensions. The back-flow integrals from O'Brien's method essentially reduce long-range interactions to being localized in extent. Their use with Ewald sums can be thought of as integrating out the average effect of far particles. The near-field lubrication interactions and the connectivity of particle clusters are unaffected by these "mean-field" effects. Thus, one can imagine simulating a system of hundreds or thousands of particles in which, as far as long-range interactions are concerned, each particle finds itself in a periodic box containing only tens of particles, but all particles may interact through the short-range forces in order to preserve large-scale connectivity. This approach produces a large, sparse matrix that is still positive-definite, symmetric, and diagonally dominant, and that optimally requires only $O(N)$ operations for solution. The procedure also naturally lends itself to multiple time stepping and iterative schemes and thus holds potential for simulating very large systems. It does, however, incorrectly replicate small-scale fluctuations through the Ewald summations and has not been tested. Only future trials will show whether or not this is a viable method.

The Stokesian-dynamics method we have developed provides a rigorous and accurate procedure for dynamically simulating hydrodynamically interacting particles and suspensions. As other researchers find applications for Stokesian dynamics in their own research, we anticipate that the method will see increasing use in the future and become a standard investigative tool, just as Monte Carlo calculations and molecular dynamics are almost routine in statistical chemistry and physics. One can even envision the day when Stokesian dynamics can be used in the design and control of particle-filled composite materials. At the same time, we hope to see a renewed effort at theory and experiment, particularly of concentrated dispersions, so that our understanding of and ability to predict suspension behavior may advance along the three fronts of theory, experiment, and computation.

\section{ACKNOWLEDGMENTS}

We would like to thank our colleagues L. Durlofsky, R. Phillips, and J. Lester, who helped develop the Stokesian-dynamics simulation method with us. Financial support for this work has come from several sources: in France through the Centre National de la Recherche Scientifique and the Centre de Calcul Vectoriel pour la Recherche, and in the United States through the Monsanto Company, the National Science Foundation (CBT-8451597, CBT-8696067, and INT-8413695), the Camille and Henry Dreyfus Foundation, and the NSF-sponsored San Diego Supercomputer Center. 


\section{Literature Cited}

Adler, P. M. 1987. Hydrodynamic properties of fractal flocs. See Faraday Discussions 1987. In press

Adler, P. M., Brenner, H. 1982. Dispersion resulting from flow through spatially periodic porous media. II. Surface and interparticle transport. Philos. Trans. R. Soc. London Ser. A 307: 149-200

Adler, P. M., Zuzovsky, M., Brenner, H. 1985. Spatially periodic suspensions of convex particles in linear shear flows. II. Rheology. Int.J. Multiphase Flow 11:387417

Alder, B. J., Wainwright, T. E. 1958. Molecular dynamics by electronic computers. In Transport Processes in Statistical Mechanics, ed. I. Prigogine, pp. 97-131. New York: Interscience. $436 \mathrm{pp}$.

Ansell, G. C., Dickinson, E. 1986a. Brownian dynamics simulation of the fragmentation of a large colloidal floc in simple shear flow. J. Colloid Interface Sci. 110 : 73-81

Ansell, G. C., Dickinson, E. 1986b. Sediment formation by Brownian dynamics simulation: effect of colloidal and hydrodynamic interactions on the sediment structure. J. Chem. Phys. 85: 4079-86

Ansell, G. C., Dickinson, E., Ludvigson, M. 1985. Brownian dynamics of colloidalaggregate rotation and dissociation in shear flow. J. Chem. Soc. Faraday Trans. 281: $1269-84$

Arp, P. A., Mason, S. G. 1977. The kinetics of flowing dispersions. VIII. Doublets of rigid spheres (theoretical). J. Colloid Interface Sci. 61: 21-43

Bacon, J., Dickinson, E., Parker, R. 1983. Simulation of particle motion and stability in concentrated dispersions. See Faraday Discussions 1983, pp. 165-78

Batchelor, G. K. 1970a. The stress system in a suspension of force-free particles. $J$. Fluid Mech. 41: 545-70

Batchelor, G. K. 1970b. Slender-body theory for particles of arbitrary cross-section in Stokes flow. J. Fluid Mech. 44: 419-40

Batchelor, G. K. 1972. Sedimentation in a dilute dispersion of spheres. J. Fluid Mech. 52: 245-68

Batchelor, G. K. 1974. Transport properties of two-phase materials with random structure. Ann. Rev. Fluid Mech. 6: 227-55

Batchelor, G. K. 1976a. Developments in microhydrodynamics. In Theoretical and Applied Mechanics, ed. W. Koiter, pp. 3355. Amsterdam: North-Holland. $260 \mathrm{pp}$.

Batchelor, G. K. 1976b. Brownian diffusion of particles with hydrodynamic interaction. J. Fluid Mech. 74: 1-29

Batchelor, G. K. 1977. The effect of Brown- ian motion on the bulk stress in a suspension of spherical particles. J. Fluid Mech. 83: 97-117

Batchelor, G. K. 1982. Sedimentation in a dilute polydisperse system of interacting spheres. Part 1. General theory. J. Fluid Mech. 119: 379-408

Batchelor, G. K. 1983. Diffusion in a dilute polydisperse system of interacting spheres. J. Fluid Mech. 131: 155-75

Batchelor, G. K., Green, J. T. 1972. The determination of the bulk stress in a suspension of spherical particles to order $c^{2}$. J. Fluid Mech. 56: 401-27

Beenakker, C. W. J. 1984. The effective viscosity of a concentrated suspension of spheres (and its relation to diffusion). Physica A 128: 48-81

Beenakker, C. W. J. 1986. Ewald sum of the Rotne-Prager tensor. J. Chem. Phys. 85: 1581-82

Beenakker, C. W. J., Mazur, P. 1983. Selfdiffusion of spheres in a concentrated suspension. Physica A 120: 388-410

Beenakker, C. W, J., Mazur, P. 1984. Diffusion in a concentrated suspension II. Physica A 126: 349-70

Blanc, R., Belzons, M., Camoin, C., Bouillot, J. L. 1983. Cluster statistics in a bidimensional suspension: comparison with percolation. Rheol. Acta 22: 505-11

Bonnecaze, R. 1987. A method for determining the effective conductivity of dispersions of particles. MS thesis. Calif. Inst. Technol., Pasadena. $31 \mathrm{pp}$.

Bossis, G., Brady, J. F. 1984. Dynamic simulation of sheared suspensions. I. General method. J. Chem. Phys. 80: 5141-54

Bossis, G., Brady, J. F. 1987. Self diffusion of Brownian particles in concentrated suspensions under shear. J. Chem. Phys. In press

Bouillot, J. L., Camoin, C., Belzons, M., Blanc, R., Guyon, E. 1982. Experiments on 2-D suspensions. Adv. Colloid Interface Sci. 17: 299-305

Brady, J. F., Bossis, G. 1985. The rheology of concentrated suspensions of spheres in simple shear flow by numerical simulation. J. Fluid Mech. 155: 105-29

Brenner, H. 1974. Rheology of a dilute suspension of axisymmetric Brownian particles. Int. J. Multiphase Flow 1: 195-341

Brenner, H. 1980a. A general theory of Taylor dispersion phenomena. PhysicoChem. Hydrodyn. 1: 91-123

Brenner, H. $1980 \mathrm{~b}$. Dispersion resulting from flow through spatially periodic porous media. Philos. Trans. R. Soc. London Ser. A 297: 81-133

Brenner, H. 1982. A general theory of Taylor 


\section{BRADY \& BOSSIS}

dispersion phenomena. II. An extension. Physico-Chem. Hydrodyn. 3: 139-57

Brenner, H., O'Neill, M. E. 1972. On the Stokes resistance of multiparticle systems in a linear shear field. Chem. Eng. Sci. 27: 1421-39

Brinkman, H. C. 1947. A calculation of the viscous force exerted by a flowing fluid on a dense swarm of particles. Appl. Sci. Res. A 1: 27-34

Camoin, C., Bossis, G., Guyon, E., Blanc, R., Brady, J. F. 1985. Suspensions modeles à deux dimensions. J. Méc. Théor Appl. Num. Spéc., pp.'141-64

Campbell, C. S., Brennen, C. E. 1985. Computer simulation of granular shear flows. J. Fluid Mech. 151: 161-88

Campbell, C. S., Gong, A. 1986. The stress tensor in a two-dimensional granular shear flow. J. Fluid Mech. 164: 107-25

Chae, D. G., Ree, F. H., Ree, J. 1969. Radial distribution functions and equation of state of the hard-disk fluid. J. Chem. Phys. 50: 1581-89

Chen, Z.-Y., Deutch, J. M., Meakin, P. 1984. Translational friction coefficient of diffusion limited aggregates. J. Chem. Phys. 80: 2982-83

Childress, S. 1972. Viscous flow past a random array of particles. J. Chem. Phys. 56 : 2527-39

Chwang, A. T., Wu, T. Y.-T. 1975. Hydromechanics of low-Reynolds-number flow. Part 2. Singularity method for Stokes flow. J. Fluid Mech. 67: 787-815

Davis, R. H., Acrivos, A. 1985. Sedimentation of noncolloidal particles at low Reynolds numbers. Ann. Rev. Fluid Mech. 17: $91-118$

de Leeuw, S. W., Perram, J. W., Smith, E. R. 1983. Simulation of electrostatic systems in periodic boundary conditions. III. Further theory and applications. Proc. $R$. Soc. London Ser. A 388: 177-93

Dickinson, E. 1983. Dispersions of interacting colloidal particles. Ann. Rep. C. R. Soc. Chem., pp. 3-37

Dickinson, E. 1985. Brownian dynamics with hydrodynamic interactions: the application to protein diffusional problems. Chem. Soc. Rev. 14: 421-55

Dickinson, E., Allison, S. A., McCammon, J. A. 1985. Brownian dynamics with rotation-translation coupling. J. Chem. Soc. Faraday Trans. 2 81: 591-601

Dotson, P. J. 1983. Brownian dynamics simulation of macromolecules in steady shear flow. J. Chem. Phys. 79: 5730-31

Durlofsky, L. 1986. Topics in fluid mechanics: I. Flow between finite rotating disks. II. Simulation of hydrodynamically interacting particles in Stokes flow. PhD thesis. Mass. Inst. Technol., Cambridge. 402 pp.
Durlofsky, L., Brady, J. F. 1987. Analysis of the Brinkman equation as a model for flow in porous media. Phys. Fluids. In press

Durlofsky, L., Brady, J. F., Bossis, G. 1987. Dynamic simulation of hydrodynamically interacting particles. J. Fluid Mech. 180: 21- 49

Einstein, A. 1906. Eine neue Bestimmung der Moleküldimensionen. Ann. Phys. 19: 289306 (34: 591-92, 1911)

Ermak, D. L., McCammon, J. A. 1978. Brownian dynamics with hydrodynamic interactions. J. Chem. Phys. 69: 135260

Evans, D. J., Hoover, W. G. 1986. Flows far from cquilibrium via molecular dynamics. Ann. Rev. Fluid Mech. 18: 243-64

Ewald, P.P. 1921. Die Berechnung optischer und elektrostatischer Gittcrpotentialc. Ann. Phys. 64: 253-87

Family, F., Landau, D. P., eds. 1984. Kinetics of Aggregation and Gelation. Amsterdam: North-Holland. $274 \mathrm{pp}$.

Faraday Discussions. 1983. Concentrated Colloidal Dispersions. Faraday Discuss. Chem. Soc. Vol. 76. 379 pp.

Faraday Discussions. 1987. Brownian Motion. Faraday Discuss. Chem. Soc. Vol. 83. In press

Fixman, M. 1987. Brownian dynamics of chain polymers. See Faraday Discussions 1987. In press

Fogelson, A. L. 1986. Numerical solution of $3 D$ Stokes' equations with suspended particles. Presented at SIAM Workshop Multiphase Flow, Leesburg, Va.

Ganatos, P., Pfeffer, R., Weinbaum, S. 1978. A numerical-solution technique for threedimensional Stokes flow, with application to the motion of strongly interacting spheres in a plane. J. Fluid Mech. 84: $79-$ 111

Gaylor, K., Snook, I., van Megen, W. 1981. Comparison of Brownian dynamics with photon correlation spectroscopy of strongly interacting colloidal particles. $J$. Chem. Phys. 75: 1682-89

Goodman, J., Hut, P., eds. 1985. Dynamics of Star Clusters. Dordrecht: Reidel. 622 pp.

Hermans, J., ed. 1984. Molecular Dynamics and Protein Structure. Western Springs, Ill: Polycrystal Book Serv. 194 pp.

Hinch, E. J. 1975. Application of the Langevin equation to fluid suspensions. $J$. Fluid Mech. 72: 499-511

Hinch, E. J. 1977. An averaged-equation approach to particle interactions in a fluid suspension. J. Fluid Mech. 83: 695-720

Hoover, W. G. 1983. Nonequilibrium molecular dynamics. Ann. Rev. Phys. Chem. 34: $103-27$

Hoover, W. G. 1986. Molecular Dynam- 
ics, Lect. Notes Phys. Vol. 258. Berlin: Springer-Verlag. $138 \mathrm{pp}$.

Howells, I. D. 1974. Drag due to the motion of a Newtonian fluid through a sparse random array of small fixed rigid objects. $J$. Fluid Mech. 64: 449-75

Jeffrey, D. J. 1973. Conduction through a random suspension of spheres. Proc. $R$. Soc. London Ser. A 335: 355-67

Jeffrey, D. J., Acrivos, A. 1976. The rheological properties of suspensions of rigid particles. AIChE J. 22: 417-32

Jeffrey, D. J., Onishi, Y. 1984. Calculation of the resistance and mobility functions for two unequal rigid spheres in low-Reynolds-number flow. J. Fluid Mech. 139: $261-90$

Karnis, A., Goldsmith, H. L., Mason, S. G. 1966. The kinetics of flowing dispersions. I. Concentrated suspensions of rigid particles. J. Colloid Interface Sci. 22: 531-53

Kim, S., Miftlin, R. T. 1985. The resistance and mobility functions of two equal spheres in low-Reynolds-number flow. Phys. Fluids 28: 2033-45

Kim, S., Russel, W. B. 1985. Modelling of porous media by renormalization of the Stokes cquations. J. Fluid Mech. 154: 26986

Koch, D. L., Brady, J. F. 1985. Dispersion in fixed beds. J. Fluid Mech. 154: 399-427

Koch, D. L., Brady, J. F. 1987. Nonlocal dispersion in porous media: nonmechanical effects. Chem. Eng. Sci. 42: 1377-92

Leighton, D., Acrivos, A. 1987. Measurement of shear induced self-diffusion in concentrated suspensions of spheres. $J$. Fluid Mech. 177: 109-31

Levesque, D., Weis, J. J., Hansen, J. P. 1986. Simulation of classical fluids. In Monte Carlo Methods in Statistical Physics, Top. Curr. Phys. ed. K. Binder, 7: 47-120. Berlin: Springer-Verlag. 410 pp. 2nd ed.

Mazur, P., van Saarloos, W. 1982. Manysphere hydrodynamic interactions and mobilities in a suspension. Physica A 115: 21-57

McCammon, J. A. 1984. Protein dynamics. Rep. Prog. Phys. 47: 1-46

Meakin, P., Deutch, J. M. 1987. Properties of the fractal measure describing the hydrodynamic force contributions for fractal aggregates moving in a quiescent fluid. J. Chem. Phys. 86: 4648-56

Meakin, P., Chan, Z.-Y., Deutch, J. M. 1985. The translational friction coefficient and time dependent cluster size distribution of three dimensional cluster-cluster aggregation. J. Chem. Phys. 82: 3786-89

Nunan, K. C., Keller, J. B., 1984. Effective
STOKESIAN DYNAMICS

viscosity of a periodic suspension. $J$. Fluid Mech. 142: 26987

O'Brien, R. W. 1979. A method for the calculation of the effective transport properties of suspensions of interacting particles. J. Fluid Mech. 56: 401-27

Rallison, J. M., Hinch, E. J. 1986. The effect of particle interactions on dynamic light scattering from a dilute suspension. $J$. Fluid Mech. 167: 131-68

Russel, W. B. 1980. Review of the role of colloidal forces in the rheology of suspensions. J. Rheol. 24: 287-317

Russel, W. B. 1981. Brownian motion of small particles suspended in liquids. Ann. Rev. Fluid Mech. 13: 425-55

Saab, H. H., Dotson, P. J. 1987. Nonequilibrium statistics of flexible macromolecules in dilute solutions. II. Macromolecular extension and comparison with nonequilibrium Brownian dynamics. $J$. Chem. Phys. 86: 3039-45

Saffman, P. G. 1973. On the settling speed of free and fixed suspensions. Stud. Appl. Math. 52: 115-27

Schowalter, W. R. 1984. Stability and coagulation of colloids in shear flows. Ann. Rev. Fluid Mech. 16: 245-61

Tam, C. K. W. 1969. The drag on a cloud of spherical particles in low Reynolds number flow. J. Fluid Mech. 38: 537-46

Theodorou, D. N., Suter, U. W. 1986. Atomistic modeling of mechanical properties of polymeric glasses. Macromolecules 19: 139-54

van Megen, W., Underwood, S. M., Snook, I. 1986. Tracer diffusion in concentrated colloidal dispersions. J. Chem. Phys. 85: 4065-72

van Saarloos, W., Mazur, P. 1983. Manysphere hydrodynamic interactions. II. Mobilities at finite frequencies. Physica $A$ 120: $77-102$

Walton, O. R., Braun, R. L. 1986. Viscosity, granular-temperature, and stress calculations for shearing assemblies of inelastic, frictional disks. J. Rheol. 30: 949-80

Walton, O. R., Hagen, D. A., Cooper, J. N. 1984. Interparticle force models for computational simulation of granular solids flow. Presented at Int. Congr. Theor. Appl. Mech., 16th, Lyngby, Den.

Zick, A., Homsy, G. M. 1982. Stokes flow through periodic arrays of spheres. $J$. Fluid Mech. 115: 13-26

Zuzovsky, M., Adler, P. M., Brenner, H. 1983. Spatially periodic suspensions of convex particles in linear shear flows. III. Dilute arrays of spheres suspended in Newtonian fluids. Phys. Fluids 26: 1714 23 
Annual Review of Fluid Mechanics
Volume 20,1988

\section{CONTENTS}

The First Turbulence Measurements: A Tribute to Hugh L.

Dryden, $A . M$. Kuethe

Fractals in Fluid Mechanics, D. L. Turcotte 5

Adaptive Wind Tunnels, W. R. Sears and J. C. Erickson, Jr. 17

Multiphase Flow in Porous Media, Pierre M. Adler and Howard $\begin{array}{ll}\text { Brenner } & 35\end{array}$

Fluid Models of Geological Hotspots, John A. Whitehead 61

Remote Sensing of the Sea Surface, O. M. Phillips 89

StokesIAN DYNAMICS, John F. Brady and Georges Bossis 111

Initial Stage of Water Impact, $A$. $A$. Korobkin and

V.V. Pukhnachov

Magnetic Fields in the Solar Convection Zone:

Magnetoconvection and Magnetic Buoyancy, D. W. Hughes and M. R. E. Proctor

Hamiltonian Fluid Mechanics, Rick Salmon 225

Surf-Zone Dynamics, J. A. Batijes 257

Sand Transport on the Continental Shelf, K. R. Dyer and

R. L. Soulsby

295

FoAm Flows, Andrew M. Kraynik

Instability Mechanisms in Shear-Flow Transition, Bruce J.

Bayly, Steven A. Orszag, and Thorwald Herbert

359

Compliant Coatings, James J. Riley, Mohamed Gad-el-Hak, and Ralph W. Metcalfe

Digital Image Processing in Flow Visualization, Lambertus Hesselink

SECONDARY INSTABILITY OF BOUNDARY LAYERS, Thorwald Herbert

INDEXES

Subject Index

Cumulative Index of Contributing Authors, Volumes 1-20 535

Cumulative Index of Chapter Titles, Volumes 1-20 